\begin{tabular}{|c|l|}
\hline Title & $\begin{array}{l}\text { Quantitative phase field modeling of nonisothermal solidification in dilute multicomponent alloys with arbitrary } \\
\text { diffusivities }\end{array}$ \\
\hline Author(s) & Ohno, Munekazu \\
\hline Citation & $\begin{array}{l}\text { Physical Review E, 86(5), 051603 } \\
\text { https://doi.org/10.1103/PhysRevE.86.051603 }\end{array}$ \\
\hline Issue Date & 2012-11 \\
\hline Doc URL & http://hdl.handle.net/2115/50824 \\
\hline Rights & O2012A A merican Physical Society \\
\hline Type & article \\
\hline File Information & PRE86-5_051603.pdf \\
\hline
\end{tabular}

Instructions for use 


\title{
Quantitative phase-field modeling of nonisothermal solidification in dilute multicomponent alloys with arbitrary diffusivities
}

\author{
Munekazu Ohno \\ Division of Materials Science and Engineering, Faculty of Engineering, Hokkaido University, Kita 13 Nishi 8, \\ Kita-ku, Sapporo, Hokkaido 060-8628, Japan
}

(Received 30 July 2012; published 8 November 2012)

\begin{abstract}
A quantitative phase-field model is developed for simulating microstructural pattern formation in nonisothermal solidification in dilute multicomponent alloys with arbitrary thermal and solutal diffusivities. By performing the matched asymptotic analysis, it is shown that the present model with antitrapping current terms reproduces the free-boundary problem of interest in the thin-interface limit. Convergence of the simulation outcome with decreasing the interface thickness is demonstrated for nonisothermal free dendritic growth in binary alloys and isothermal and nonisothermal free dendritic growth in a ternary alloy.
\end{abstract}

DOI: 10.1103/PhysRevE.86.051603

PACS number(s): 81.10.-h, 64.70.D-, 68.08.-p

\section{INTRODUCTION}

Control of solidification microstructure is an important issue in both design and production of alloys. Most of practical alloys are multicomponent systems and therefore analyses and prediction of the solidification microstructure require coupled multicomponent diffusion to be precisely described. Furthermore, the influence of heat diffusion on microstructural evolution is not negligible, especially in dilute alloys such as a number of practical carbon steels.

The phase-field model is a powerful tool to describe microstructural pattern formation during solidification [1-4]. This is the diffuse interface approach in which a steep but smooth variation of the order parameter called the phase-field characterizes the interface region. The chief advantage of this approach is that one can avoid tracking the sharp interface in complex microstructural patterns. The phase-field model has been applied to a variety of solidification processes [1-4] and its capability of affording qualitative understanding of phenomena is generally acknowledged. Despite the tremendous success, however, a longstanding issue remains unresolved regarding the quantitative aspect. This issue is intimately connected to the diffuse interface concept and its phenomenological nature.

Most of the phase-field models for solidification are essentially phenomenological in that they are not derived based on a coarse-graining procedure from atomistic description of phenomena. The models should be asymptotically mapped onto the sharp-interface equations in order to be made quantitatively meaningful. Early models have been developed so as to reproduce the free-boundary problem of interest in the so-called sharp-interface limit where the interface thickness $W$ is approximated to zero [5,6]. However, it is prerequisite for this diffuse interface approach to employ a finite value of $W$ in practice. A typical value of $W$ for the solid-liquid interface is of the order of a few nm according to atomistic simulations [7]; thus, it needs a mesh size of $\AA$ order to describe continuous variations of physical quantities inside the interface region. This limits the system size to extremely small, making it impossible to deal with problems on the microstructural scale. A similar restriction is also imposed on the time scale. Therefore, one has to increase $W$ by orders of magnitude from the realistic thickness. This increment, in turn, unrealistically magnifies some physical effects, the contributions of which scale with $W$, and it accordingly precludes quantitatively correct simulations.

Karma and Rappel put forward a new procedure to resolve this impasse, which is called the thin-interface limit [8,9]. In this limit, $W$ is taken to be smaller than any physical length appearing on the microstructural scale but is much larger than the realistic thickness. They developed a symmetric model for a pure substance (equal thermal diffusivities in the solid and liquid) based on the thin-interface limit. In their model, the free-boundary problem is recovered for a finite value of $W$. This method has opened the way for quantitatively correct simulations. However, a simple extension of the thin-interface limit to the case of arbitrary diffusivities was found to involve difficulties [10]. To be more specific, several interface effects emerge in the thin-interface limit, which cannot readily be eliminated within traditional forms of the model. This problem was overcome by introducing an additional solute current termed the antitrapping current into the solute diffusion equation [11]. The antitrapping current offers an additional degree of freedom in modeling, making it possible to remove the anomalous interface effects and thus to conduct quantitatively correct simulations. The antitrapping current approach was first developed for alloy solidification in a dilute binary alloy with zero diffusivity in the solid (one-sided model) $[11,12]$. It was extended to describe multiphase solidification in a binary alloy with zero diffusivities in the solids (one-sided model) [13] and alloy solidification with coupled heat and solute diffusion in a dilute binary alloy having zero solutal diffusivity in the solid and equal thermal diffusivities in the solid and liquid (one-sided solute transport and symmetric heat transport) [14]. Moreover, it was extended to deal with isothermal solidification in multicomponent alloys with zero solutal diffusivities in the solid (one-sided model) [15]. These models are being increasingly utilized for quantitative simulations of solidification phenomena [16-34].

It should be noted that the thin-interface asymptotics, which is indispensable to the development of quantitative models, has so far been carried out only for some specific systems [8-14]. An important step toward further generalization is to model, on the basis of the asymptotics, alloy solidification involving coupled heat and solute diffusion in multicomponent alloys. 
This is tackled in this study. Of particular interest here is the case of arbitrary values of solutal and thermal diffusivities. The antitrapping current approach was recently extended to treat arbitrary values of solutal diffusivity in the solid $[4,35,36]$. In this study, the method proposed by the present authors for isothermal solidification in a binary alloy [36] is extended to model nonisothermal solidification in multicomponent alloys. As pointed out in Ref. [37] and discussed later, the antitrapping current approach, by its nature, cannot completely solve all the problems in the two-sided model. However, the present approach can be validated as long as the interface velocity is finite. By performing a matched asymptotic analysis, it is demonstrated that the present model reproduces the freeboundary problem of interest in the thin-interface limit. The validity and efficiency of the model are investigated by means of numerical simulations. Reasonable convergence behavior of the simulation outcome with decrease in $W$ is shown for nonisothermal free dendritic growth in binary alloys and isothermal and nonisothermal free dendritic growth in a ternary alloy.

The organization of this paper is as follows. In Sec. II, the sharp-interface model for nonisothermal solidification in multicomponent alloys is described. The phase-field model is developed in Sec. III and the matched asymptotic analysis is given in Sec. IV, where some issues on the modeling, such as inclusion of dependence of diffusivities on local temperature and concentrations, are discussed. The numerical tests are presented in Sec. V, followed by the summary in Sec. VI.

\section{SHARP-INTERFACE MODEL}

The sharp-interface equations of interest are explained. In the next section, the phase-field model is developed in such a way that the sharp-interface equations are reproduced in the thin-interface limit. Hence, the sharp-interface description given in this section exactly specifies the physical system to which the present phase-field model is applicable.

We focus on nonisothermal solidification in a dilute alloy with $N$ 's components (solvent and $N-1$ 's solute atoms). The heat and solute diffusion equations in the bulk phases are, respectively, given by

$$
\begin{gathered}
\partial_{t} T=\nabla D_{v, T}\left(T,\left\{c_{i}\right\}\right) \nabla T, \\
\partial_{t} c_{i}=\nabla D_{v, i}\left(T,\left\{c_{i}\right\}\right) \nabla c_{i} \quad \text { for } \quad i=1,2, \ldots, N-1,
\end{gathered}
$$

where $\partial_{t}$ represents the time derivative, $T$ is the temperature, $D_{v, T}$ is the thermal diffusivity in $v$ phase with $v=l$ for liquid and $v=s$ for solid, $c_{i}$ is the concentration of solute $i$ atoms, and $D_{v, i}$ is the solutal diffusivity of $i$ atoms in $v$ phase. The specific heat per unit volume $c_{p}$ in the solid is assumed to be equal to that in the liquid in Eq. (2.1). However, we consider the difference in the thermal conductivity between the phases and the thermal diffusivity accordingly depends on the type of phase. Also, the dependence of the thermal and solutal diffusivities on $T$ and $\left\{c_{i}\right\}$ is taken into account in this study. For the sake of simplicity, the effects of off-diagonal elements in diffusivity matrix are ignored.
The temperature at the interface $T^{*}$ obeys the GibbsThomson relation,

$$
T^{*}=T_{m}+\sum_{i}^{N-1} m_{i} c_{l, i}^{*}-\Gamma \kappa-V_{n} / \mu_{k},
$$

where $T_{m}$ is the melting temperature of pure solvent, $m_{i}$ is the liquidus slope of $i$ atoms, $c_{l, i}^{*}$ is the liquid concentration of $i$ atoms at the interface, $\Gamma$ is the Gibbs-Thomson coefficient, $\kappa$ is the interface curvature, $V_{n}$ is the normal velocity of interface, and $\mu_{k}$ is the linear kinetic coefficient. The interface properties are taken to be isotropic and the anisotropy will be considered later. In addition, the energy and mass conservation laws are satisfied at the interface,

$$
\begin{aligned}
\left(L / c_{p}\right) V_{n} & =\left.D_{s, T} \partial_{n} T\right|^{-}-\left.D_{l, T} \partial_{n} T\right|^{+}, \\
c_{l, i}^{*}\left(1-k_{i}\right) V_{n} & =\left.D_{s, i} \partial_{n} c_{i}\right|^{-}-\left.D_{l, i} \partial_{n} c_{i}\right|^{+} \text {for } \\
i & =1,2, \ldots, N-1,
\end{aligned}
$$

where $L$ is the latent heat per unit volume and $k_{i}$ is the partition coefficient of $i$ atoms. $\left.\partial_{n} T\right|^{+}\left(\left.\partial_{n} T\right|^{-}\right)$in Eq. (2.4) is the spatial derivative of $T$ normal to the interface taken on the liquid (solid) side of the interface and the same notation is used for the spatial derivative of $c_{i}$ in Eq. (2.5).

For convenience, we introduce the dimensionless undercooling (temperature) and supersaturation (concentration) with respect to an equilibrium state $\left(\left\{c_{l, i}^{e}\right\}, T_{0}\right)$, where $c_{l, i}^{e}$ is the equilibrium liquid concentration of $i$ atoms at $T_{0}$ and thus $T_{0}=T_{m}+\sum m_{i} c_{l, i}^{e}$. These dimensionless variables are defined as

$$
\begin{gathered}
\theta=\frac{T-T_{0}}{L / c_{p}}, \\
u_{i}=\frac{c_{i}-c_{v, i}^{e}}{z_{v, i}\left(1-k_{i}\right) c_{l, i}^{e}},
\end{gathered}
$$

where $z_{l, i}=1$ in the liquid and $z_{s, i}=k_{i}$ in the solid. It is noted that $u_{i}$ is continuous at the interface. Using these variables, Eqs. (2.1)-(2.5) are rewritten as

$$
\begin{gathered}
\partial_{t} u_{i}=\nabla D_{v, i} \nabla u_{i} \quad \text { for } \quad i=1,2, \ldots, N, \\
\sum_{i=1}^{N} M_{i} u_{i}^{*}=-d_{0} \kappa-\beta V_{n}, \\
{\left[1+\left(1-k_{i}\right) u_{i}^{*}\right] V_{n}=\left.k_{i} D_{s, i} \partial_{n} u_{i}\right|^{-}-\left.D_{l, i} \partial_{n} u_{i}\right|^{+} \text {for }} \\
i=1,2, \ldots, N,
\end{gathered}
$$

where $d_{0}=\Gamma /\left(L / c_{p}\right)$ is the thermal capillary length and $M_{i}=d_{0} / d_{i}$ is a ratio of the thermal capillary length to the chemical capillary length associated with $i$ atoms, $d_{i}=-\Gamma /\left[m_{i}\left(1-k_{i}\right) c_{l, i}^{e}\right] . \beta$ is given by $\beta=c_{p} /\left(\mu_{k} L\right)$. In Eqs. (2.8)-(2.10), $\theta$ is rewritten as $\theta=u_{N}$ and the related parameters are accordingly given by $D_{v, N}=D_{v, T}, k_{N}=$ 1 , and $M_{N}=1$. Therefore, the nonisothermal solidification process in $N$ 's component alloys can be regarded as the isothermal solidification process in $N+1$ 's component alloys. This is a natural consequence of the analogy existing between thermal and solutal problems [38]. Equations (2.8)-(2.10) specify the free-boundary problem of interest. In the next section, the phase-field model is developed so as to reproduce 
the sharp-interface equations (2.8)-(2.10) in the thin-interface limit.

\section{PHASE-FIELD MODEL}

Several phase-field models were developed to describe multicomponent alloys [39-42] and they were basically formulated in a variational framework. The phase-field model put forward in this paper is an extension of the model for isothermal solidification in a binary alloy [36] to the case of nonisothermal solidification in multicomponent alloys. This corresponds to a nonvariational formulation and the main concern here is the final form of the time evolution equations made being consistent with the sharp-interface model. In this regard, the detail on physical assumptions made in the formulation may be of secondary importance. The variational derivation of the phase-field model for nonisothermal solidification in multicomponent alloys is detailed in the Appendix and the final form of the quantitative phase-field model is briefly described in this section.

Before going into the detail, an issue on coupling between bulk and interface properties is briefly discussed. In early alloy models, coupling between bulk and interface properties occurs in the interface region, which provokes a variation of the interfacial property with the concentration in the interface and makes simulation results dependent on $W$. A way out of this situation is to define the interface region as a mixture of two phases, each of which possesses each thermodynamic property inside the interface $[43,44]$. In addition, a new and different approach was recently proposed based on a variational formulation from a grand-potential functional $[45,46]$. In this study, we employ one of the former models, called the Kim-Kim-Suzuki (KKS) model [43]. This model allows the equilibrium phase-field profile to be decoupled from the concentration profile and the interface property becomes independent of the concentration profile.

As in the previous section, we consider a dilute alloy consisting of $N-1$ 's solutes. We introduce the phase-field $\phi$ which takes $\phi=+1$ in the solid and $\phi=-1$ in the liquid and continuously changes from +1 to -1 inside the interface region. Based on the KKS model [43], the time evolution equation of $\phi$ is given as

$$
\hat{M}_{\phi}^{-1} \partial_{t} \phi=\sigma^{2} \nabla^{2} \phi-\omega f_{d w}^{\prime}-\frac{1}{2} g^{\prime}(\phi) \Delta f_{\text {driv }}^{*},
$$

where $\hat{M}_{\phi}$ is the phase-field mobility and $\sigma$ and $\omega$ are constants. The interfacial energy is isotropic in this formation and the anisotropic effect is discussed later. $f_{d w}(\phi)$ is a double-well potential with minima at $\phi= \pm 1$ and $g(\phi)$ is an interpolating function that satisfies $g( \pm 1)= \pm 1$ and $g^{\prime}( \pm 1)=0 . \Delta f_{\text {driv }}^{*}$ corresponds to the driving force of solidification and, as detailed in the Appendix, it is given for dilute alloys by

$$
\begin{aligned}
\Delta f_{\text {driv }}^{*}= & \left(L / T_{m}\right)\left(T-T_{0}\right) \\
& +\left(R T_{m} / v_{m}\right) \sum_{i=1}^{N-1}\left(1-k_{i}\right)\left(c_{l, i}-c_{l, i}^{e}\right),
\end{aligned}
$$

where $R$ is the gas constant and $v_{m}$ is the molar volume. The other parameters in Eq. (3.2) are the same as those employed in Sec. II. It is noted that concentration of $i$ atoms in the liquid state $c_{l, i}$ is defined even in the solid region within the
KKS model and it is related to the concentration of solid $c_{s, i}$ through the condition of equal chemical potential. In the dilute solution, the relation $c_{s, i}=k_{i} \mathrm{c}_{l, i}$ is satisfied.

The solute diffusion equation is expressed as

$$
\partial_{t} c_{i}=\nabla D_{i}(\phi) \nabla c_{l, i}
$$

where $D_{i}(\phi)$ is function of $\phi$ that satisfies $D_{i}(+1)=k_{i} \mathrm{D}_{s, i}$ and $D_{i}(-1)=D_{l, i}$. Although not explicitly specified for convenience, $D_{s, i}, D_{l, i}$, and thus $D_{i}(\phi)$ depend on the concentrations and temperature. The time evolution of temperature is described by the following equation:

$$
\partial_{t} T=\nabla\left[D_{T}(\phi) \nabla T\right]+\frac{L}{c_{p}} \frac{\partial_{t} g(\phi)}{2},
$$

where $D_{T}(\phi)$ is the thermal diffusivity which satisfies $D_{T}(+1)$ $=D_{s, T}$ and $D_{T}(-1)=D_{l, T}$. The details of the derivations of Eqs. (3.3) and (3.4) are described in the Appendix.

The time evolution of the system can be described by solving a set of time evolution equations (3.1), (3.3), and (3.4). However, this model is plagued by the anomalous interface effects that preclude the quantitatively correct simulations [10]. These effects include concentration and temperature jumps across the interface, associated with solute trapping and thermal trapping [47], interface stretching and surface diffusion corrections to heat and mass conservation laws at the interface. To eliminate the anomalous effects, the additional fluxes are introduced into the solute and heat transport equations as follows:

$$
\begin{gathered}
\partial_{t} c_{i}=\nabla\left[D_{i}(\phi) \nabla c_{l, i}-\vec{J}_{A T, i}\right] \text { for } i=1,2, \ldots, N-1, \\
\partial_{t} T=\nabla\left[D_{T}(\phi) \nabla T-\vec{J}_{A T, T}\right]+\frac{L}{c_{p}} \frac{\partial_{t} h(\phi)}{2}
\end{gathered}
$$

where $\vec{J}_{A T, i}$ is called the solutal antitrapping current $[11,12]$ and $\vec{J}_{A T, T}$ is called the thermal antitrapping current in this paper. These terms are given by

$$
\begin{gathered}
\vec{J}_{A T, i}=a_{i}(\phi) \frac{\sigma}{\sqrt{\omega}}\left(c_{l, i}-c_{s, i}\right) \partial_{t} \phi \vec{n}, \\
\vec{J}_{A T, T}=a_{T}(\phi) \frac{\sigma}{\sqrt{\omega}} \frac{L}{c_{p}} \partial_{t} \phi \vec{n},
\end{gathered}
$$

where $\vec{n}=-\nabla \phi /|\nabla \phi|$ is the unit vector normal to the interface. $a_{i}(\phi)$ and $a_{T}(\phi)$ are new interpolating functions, whose forms depend on the definition of the interpolating functions. For a set of the interpolating functions described later, one obtains

$$
\begin{aligned}
& a_{i}(\phi)=\frac{1}{2 \sqrt{2}}\left(1-\frac{k_{i} D_{s, i}}{D_{l, i}}\right) \psi_{i}, \\
& a_{T}(\phi)=\frac{1}{2 \sqrt{2}}\left(1-\frac{D_{s, T}}{D_{l, T}}\right) \psi_{T},
\end{aligned}
$$

where $\psi_{i}$ and $\psi_{T}$ are parameters controlling the convergence behavior in this model and the physical meaning is explained later. These constants become 1 for one-sided case (viz., $\left.D_{s, i}=0\right)$ and symmetric case $\left(k_{i} \mathrm{D}_{s, i}=D_{l, i}\right)$. In Eq. (3.6), the function $g(\phi)$ is replaced by a new interpolating function, $h(\phi)$, $[h( \pm 1)= \pm 1]$. The form of this new function is chosen in terms of the computational efficiency. Although not explicitly 
described, we also employ $h(\phi)$ for the concentration field, that is, $c_{i}=[1+h(\phi)] c_{s, i} / 2+[1-h(\phi)] c_{l, i} / 2$.

As demonstrated in Sec. IV, this model, viz., a set of Eqs. (3.1), (3.5), and (3.6), is reduced to the sharp-interface equations in the thin-interface limit by appropriately defining the interpolating functions. In the present simulations, we used $f_{d w}(\phi)=\left(1-2 \phi^{2}+\phi^{4}\right) / 4, h(\phi)=\phi$ and $g(\phi)=$ $(15 / 8)\left[\phi-(2 / 3) \phi^{3}+\phi^{5} / 5\right]$, and $D_{i}(\phi)=\left[k_{i} \mathrm{D}_{s, i}+\right.$ $\left.D_{l, i}+\left(k_{i} \mathrm{D}_{s, i}-D_{l, i}\right) \phi\right] / 2$. When the interface kinetic effect is negligibly small, the phase-field mobility is given by

$$
\hat{M}_{\phi}=\left\{\frac{a_{2}}{J} \frac{\sigma^{2}}{\omega}\left[\frac{L^{2}}{c_{p} T_{m}} \frac{\psi_{T}}{D_{l, T}}+\frac{R T_{m}}{v_{m}} \sum_{i=1}^{N-1} \frac{\left(1-k_{i}\right)^{2} c_{l, i} \psi_{i}}{D_{l, i}}\right]\right\}^{-1},
$$

where $a_{2}$ and $J$ are numerical constants and they are given by $a_{2}=0.6278 \cdots$ and $J=16 / 15$ for a set of interpolating functions described above.

This model is applicable to nonisothermal solidification in dilute multicomponent alloys with arbitrary diffusivities. Importantly, this model contains the previously developed models $[9,11,12,14,15,36]$ as special cases. For solidification in a pure material with equal thermal diffusivities in the solid and liquid, viz., $N=1$ and $D_{s, T}=D_{l, T}\left(\Psi_{T}=1\right)$, the present model becomes identical to the symmetric model in Ref. [9]. For the isothermal solidification in a binary alloy, $N=2$, $D_{l, T}=D_{s, T}=\infty$, and $T=$ const., this model is exactly reduced to the model developed in Ref. [36] and it is further reduced to the one-sided model $[11,12]$ by setting $D_{s, 1}=0$ and $\Psi_{1}=1$. The model for coupled heat and solute diffusion in a binary alloy in Ref. [14] is reproduced by setting $N=2$, $D_{s, 1}=0, D_{s, T}=D_{l, T}$, and $\Psi_{T}=\Psi_{1}=1$. Moreover, when the one-sided problem in a multicomponent alloy is considered, the present model is consistent with the one given in Ref. [15] except that our model is limited to the dilute solution and omits the off-diagonal terms of the diffusivity matrix. Hence, the present model enables quantitative descriptions of wide range of single phase solidification phenomena. In Sec. IV, we conduct the matched asymptotic analysis to demonstrate the validity of this model. In this work all the simulations are performed for the case of symmetric heat transport, that is, $D_{s, T}=D_{l, T}$. Hence, the present simulations do not actually employ the thermal antitrapping current. However, for completeness, this term is included in the asymptotic analysis in the next section.

\section{THIN-INTERFACE ASYMPTOTICS}

\section{A. Dimensionless forms}

It is convenient to rewrite the phase-field equations in the dimensionless forms. We introduce the dimensionless variables,

$$
\begin{gathered}
\theta=\frac{T-T_{0}}{L / c_{p}}, \\
u_{i}=\frac{c_{l, i}-c_{l, i}^{e}}{\left(1-k_{i}\right) c_{l, i}^{e}} .
\end{gathered}
$$

Using these variables, the time evolution equations (3.1), (3.5), and (3.6) are rewritten as

$$
\begin{aligned}
& \tau \partial_{t} \phi= W^{2} \nabla^{2} \phi-f_{d w}^{\prime}-\hat{g}^{\prime}(\phi) \lambda\left(\theta+\sum_{i=1}^{N-1} M_{i} u_{i}\right), \\
& \frac{1}{2}\left[1+k_{i}-\left(1-k_{i}\right) h(\phi)\right] \partial_{t} u_{i} \\
&=\nabla\left\{D_{i}(\phi) \nabla u_{i}-a_{i} W\left[1+\left(1-k_{i}\right) u_{i}\right] \partial_{t} \phi \vec{n}\right\} \\
&+\frac{1}{2}\left[1+\left(1-k_{i}\right) u_{i}\right] \partial_{t} h(\phi) \text { for } i=1,2, \ldots, N-1,
\end{aligned}
$$

$$
\partial_{t} \theta=\nabla\left[D_{T}(\phi) \nabla \theta-a_{T} W \partial_{t} \phi \vec{n}\right]+\frac{1}{2} \partial_{t} h(\phi) .
$$

Here, $\tau=\left(\hat{M}_{\phi} \omega\right)^{-1}, W^{2}=\sigma^{2} / \omega, \hat{g}^{\prime}(\phi)=(J / 2) g^{\prime}(\phi), \lambda=$ $a_{1} W / d_{0}$, and $a_{1}=I / J$ with $I=2 \sqrt{2} / 3$ and $J=16 / 15 . M_{i}$ in Eq. (4.3) is the ratio of the thermal capillary length to the chemical capillary length of $i$ atoms as given in Eq. (2.9).

For the asymptotic analysis, the length and time scales are measured in unit of $d_{0}$ and $d_{0}^{2} / D_{l, \text { ref }}$, respectively, where $D_{l, \text { ref }}$ represents the lowest diffusivity in the liquid. The nondimensional forms of the evolution equations are given by

$$
\begin{aligned}
& \hat{\alpha} \varepsilon^{2} \partial_{t} \phi=\varepsilon^{2} \nabla^{2} \phi-f_{d w}^{\prime}-\varepsilon a_{1} \hat{g}^{\prime}(\phi)\left(\sum_{i=1}^{N} M_{i} u_{i}\right), \\
& \frac{1}{2}\left[1+k_{i}-\left(1-k_{i}\right) h(\phi)\right] \partial_{t} u_{i} \\
&=\nabla\left\{q_{i}(\phi) \nabla u_{i}-\varepsilon a_{i}(\phi)\left[1+\left(1-k_{i}\right) u_{i}\right] \partial_{t} \phi \vec{n}\right\} \\
& \quad+\frac{1}{2}\left[1+\left(1-k_{i}\right) u_{i}\right] \partial_{t} h(\phi) \text { for } i=1,2, \ldots, N,
\end{aligned}
$$

where $\varepsilon=W / d_{0}, \hat{\alpha}=D_{l, \text { ref }} \tau / W^{2}$, and $q_{i}(\phi)=D_{i}(\phi) / D_{l \text {,ref }}$. In these expressions, $\theta$ is denoted as $\theta=u_{N}$ and the related constants are given by $k_{N}=1, M_{N}=1, a_{N}(\phi)=a_{T}(\phi)$, and $q_{N}(\phi)=D_{T}(\phi) / D_{l, \text { ref }}$. As described in Sec. II, therefore, the nonisothermal solidification in $N$ 's component alloys can be seen as the isothermal solidification in $N+1$ 's component alloys.

The sharp-interface equations (2.8)-(2.10) are also transformed into the dimensionless forms,

$$
\begin{gathered}
\partial_{t} u_{i}=\nabla q_{v, i} \nabla u_{i} \quad \text { for } \quad i=1,2, \ldots, N \\
\sum_{i=1}^{N} M_{i} u_{i}^{*}=-\tilde{\kappa}-\tilde{\beta} v_{n} \\
{\left[1+\left(1-k_{i}\right) u_{i}^{*}\right] v_{n}=\left.q_{i}(+1) \partial_{n} u_{i}\right|^{-}-\left.q_{i}(-1) \partial_{n} u_{i}\right|^{+}} \\
i=1,2, \ldots, N .
\end{gathered}
$$

Here $q_{s, i}=q_{i}(+1) / k_{i}$ and $q_{l, i}=q_{i}(-1), v_{n}=d_{0} V_{n} / D_{l, \text { ref }}$, $\tilde{\kappa}=d_{0} \kappa$, and $\tilde{\beta}=\beta D_{l, \text { ref }} / d_{0}$. It is noted that although not explicitly specified, $q_{i}(\phi)$ depends on $\left\{u_{i}\right\}$ in the discussions given above, namely $q_{i}\left(\phi,\left\{u_{i}\right\}\right)$. In the asymptotic analysis in Sec. IV B, this dependence is first neglected for simplicity and is taken into account later. 


\section{B. Matched asymptotic analysis}

The asymptotic analysis of Eqs. (4.6) and (4.7) is carried out by expanding the solutions in powers of $\varepsilon$, which yields all important correction terms at second order [12]. It is noted that there are $N$ 's diffusion fields in this analysis but these fields are not directly coupled in the diffusion equations. The coupling of the diffusion fields occurs through the evolution of $\phi$ field. This feature makes the thin-interface limit analysis of this model similar to that for the isothermal case in a binary alloy [36]. The essential difference appears in the driving force term of the $\phi$ equation. Since full details of the analysis require lengthy discussions, most of which are the same as those given in Ref. [36], we briefly explain only the important points in the present case.

The solutions of the time evolution equations (4.6) and (4.7) are analyzed perturbatively by expanding them in an inner region and outer region in powers of $\varepsilon$. The inner region is the diffuse interface region where $\phi$ varies rapidly and the outer region is the bulk region away from the interface. The outer and inner expansions of phase field are denoted by $\Phi=\Phi_{0}+\varepsilon \Phi_{1}+\varepsilon^{2} \Phi_{2}+\cdots$ and $\phi=\phi_{0}+\varepsilon \phi_{1}+\varepsilon^{2} \phi_{2}$ $+\cdots$, respectively. Similarly, the outer and inner expansions of diffusion fields are given by $U_{i}=U_{i, 0}+\varepsilon U_{i, 1}+\varepsilon^{2} U_{i, 2}+\cdots$ and $u_{i}=u_{i, 0}+\varepsilon u_{i, 1}+\varepsilon^{2} u_{i, 2}+\cdots$, respectively. These are substituted into Eqs. (4.6) and (4.7). The expanded equations for the outer fields are readily analyzed. Since $\Phi$ is constant in the outer region, $\Phi= \pm 1$ (step function) are stable solutions for Eq. (4.6). $U_{i}$ accordingly obeys the usual diffusion equation expressed by Eq. (4.8).

In order to find the inner solution, Eqs. (4.6) and (4.7) are rewritten in terms of a local orthogonal set of curvilinear coordinates, signed distance to $\phi=0$ level set $r$, and arclength along the interface $s$. Also, the rescaled coordinate $\eta=r / \varepsilon$ is defined. Then, Eqs. (4.6) and (4.7) are expressed in terms of $\eta$ and $s$. The expansions in the inner region are matched order by order in power of $\varepsilon$ to those in the outer region based on the matching conditions. The matching conditions are the same as those given by Eqs. (4.10) and (4.11) of Ref. [36] except that $u_{n}(\eta, s)$ and $U_{n}(s)$ are replaced by $u_{i, n}(\eta, s)$ and $U_{i, n}(s)$, respectively.

By repeating the procedure described in Refs. [12,36], one can find for the solution of Eq. (4.6) at leading order $\phi_{0}(\eta)=$ $-\tanh (\eta / \sqrt{2})$ and for that of Eq. (4.7) $u_{i, 0}=\bar{U}_{i, 0}(s) \forall i$. It is noted that this solution for $\phi_{0}(\eta)$ is valid for $f_{d w}^{\prime}=-\phi+\phi^{3}$. The phase-field equation at the first order in $\varepsilon$ yields the relation

$$
\sum_{i}^{N} M_{i} \bar{U}_{i, 0}=-\tilde{\kappa}-\hat{\alpha} v_{n}
$$

This is equivalent to the Gibbs-Thomson relation when $\hat{\alpha}=\tilde{\beta}$ holds. The diffusion equation at $\varepsilon^{-1}$ order is given by

$$
\partial_{\eta}\left[q_{i}\left(\phi_{0}\right) \partial_{\eta} u_{i, 1}\right]=\frac{1}{2} \hat{v}_{n, i} \partial_{\eta} h\left(\phi_{0}\right)-\hat{v}_{n, i} \partial_{\eta}\left[a_{i}\left(\phi_{0}\right) \partial_{\eta} \phi_{0}\right],
$$

with $\hat{v}_{n, i}=v_{n}\left[1+\left(1-k_{i}\right) \bar{U}_{i, 0}\right]$. Integrating this equation once, one obtains

$$
q_{i}\left(\phi_{0}\right) \partial_{\eta} u_{i, 1}=\frac{1}{2} \hat{v}_{n, i}\left\{h\left(\phi_{0}\right)-2\left[a_{i}\left(\phi_{0}\right) \partial_{\eta} \phi_{0}\right]\right\}+A_{i, 1}(s),
$$

where $A_{i, 1}(s)$ is an integral constant. By applying the matching condition, $\lim _{\eta \rightarrow \pm \infty} \partial_{\eta} \mathrm{u}_{i, 1}=\left.\partial_{r} \mathrm{U}_{i, 0}\right|^{ \pm}$, to Eq. (4.13) and by taking into account that $a_{i}\left(\phi_{0}\right) \partial_{\eta} \phi_{0}$ term vanishes in the limit of $\eta \rightarrow \pm \infty$, one can find the relation $\left.q_{i}(\mp 1) \partial_{r} U_{i, 0}\right|^{ \pm}=$ $\mp \frac{1}{2} \hat{v}_{n, i}+A_{i, 1}(s)$, which yields

$$
\left[1+\left(1-k_{i}\right) \bar{U}_{i, 0}\right] v_{n}=\left.q_{i}(+1) \partial_{r} U_{i, 0}\right|^{-}-\left.q_{i}(-1) \partial_{r} U_{i, 0}\right|^{+} .
$$

This is the conservation law at the interface given by Eq. (4.10).

The difficulty arises in satisfying the continuity of diffusion fields at the interface. The expression for $u_{i, 1}$ is obtained by dividing both sides of Eq. (4.13) by $q_{i}\left(\phi_{0}\right)$ and by integrating that equation as follows:

$$
\begin{aligned}
u_{i, 1}= & \frac{1}{2} \hat{v}_{n, i} \int_{0}^{\eta} q_{i}\left(\phi_{0}\right)^{-1}\left\{h\left(\phi_{0}\right)-2\left[a_{i}\left(\phi_{0}\right) \partial_{\eta} \phi_{0}\right]\right\} d \xi \\
& +A_{i, 1}(s) \int_{0}^{\eta} q_{i}\left(\phi_{0}\right)^{-1} d \xi+A_{i, 2}(s),
\end{aligned}
$$

where $A_{i, 2}(s)$ is an integral constant. Then, by applying the matching condition, $\lim _{\eta \rightarrow \pm \infty}\left[u_{i, 1}-\left(\left.U_{i, 1}\right|^{ \pm}+\left.\eta \partial_{r} \mathrm{U}_{i, 0}\right|^{ \pm}\right)\right]$ $=0$ to above equation, one finds that jumps of $\left\{u_{i}\right\}$ fields generally exist at the interface, that is, $\left.U_{i, 1}\right|^{+} \neq\left. U_{i, 1}\right|^{-}$. In order for the diffusion fields to be continuous at the interface $\left(\left.U_{i, 1}\right|^{+}=\left.U_{i, 1}\right|^{-}\right)$, the following relations should be satisfied:,

$$
\begin{aligned}
& \int_{0}^{+\infty}\left\{\frac{\left[h\left(\phi_{0}\right)-2 a_{i}\left(\phi_{0}\right) \partial_{\eta} \phi_{0}\right]}{q_{i}\left(\phi_{0}\right)}-\frac{h(-1)}{q_{i}(-1)}\right\} d \eta \\
& =\int_{0}^{-\infty}\left\{\frac{\left[h\left(\phi_{0}\right)-2 a_{i}\left(\phi_{0}\right) \partial_{\eta} \phi_{0}\right]}{q_{i}\left(\phi_{0}\right)}-\frac{h(+1)}{q_{i}(+1)}\right\} d \eta, \quad \text { (4.16) } \\
& \int_{0}^{+\infty}\left[\frac{1}{q_{i}\left(\phi_{0}\right)}-\frac{1}{q_{i}(-1)}\right] d \eta=\int_{0}^{-\infty}\left[\frac{1}{q_{i}\left(\phi_{0}\right)}-\frac{1}{q_{i}(+1)}\right] d \eta .
\end{aligned}
$$

The interpolating functions, $h(\phi), a_{i}(\phi)$, and $q_{i}(\phi)$ should be determined so as to meet these requirements. However, these are not the only constraints in this model. From the $\phi$ equation at $\varepsilon^{2}$ order, one finds that the Gibbs-Thomson expression at the first order in $\varepsilon$ contains an excess term. This excess term is removed only when the relation

$$
\int_{0}^{+\infty}\left[\frac{\hat{g}\left(\phi_{0}\right)}{q_{i}\left(\phi_{0}\right)}-\frac{\hat{g}(-1)}{q_{i}(-1)}\right] d \eta=\int_{0}^{-\infty}\left[\frac{\hat{g}\left(\phi_{0}\right)}{q_{i}\left(\phi_{0}\right)}-\frac{\hat{g}(+1)}{q_{i}(+1)}\right] d \eta
$$

holds. Moreover, from $u$ equation at $\varepsilon^{0}$, the conservation law for the outer fields to first order is found to be accompanied by the excess correction terms. They have to be removed by satisfying the relations

$$
\begin{gathered}
\int_{0}^{+\infty}\left[h\left(\phi_{0}\right)-h(-1)\right] d \eta=\int_{0}^{-\infty}\left[h\left(\phi_{0}\right)-h(+1)\right] d \eta \\
\int_{0}^{+\infty}\left[q_{i}\left(\phi_{0}\right)-q_{i}(-1)\right] d \eta=\int_{0}^{-\infty}\left[q_{i}\left(\phi_{0}\right)-q_{i}(+1)\right] d \eta
\end{gathered}
$$

The elimination of anomalous interface effects requires these requirements [Eqs. (4.16)-(4.20)] to be satisfied simultaneously. 
In the symmetric model, viz., $q_{i}(+1)=q_{i}(-1)$ and thus $q_{i}(\phi)=$ const., the anomalous interface effects vanish without the antitrapping current terms $\left[a_{i}(\phi)=0\right]$ when $\hat{g}(\phi)$ and $h(\phi)$ are taken to be odd functions of $\phi$. In the one-sided model $\left[q_{i}(+1)=0\right]$, the constraints given by Eqs. (4.17) and (4.18), which are associated with the constant $A_{i, 1}(s)$, do not exist. Thanks to the additional degree of freedom fed by the antitrapping current term $a_{i}(\phi)$, three constraints-Eq. (4.16) with a slight modification and Eqs. (4.19) and (4.20) — can be simultaneously satisfied. For instance, a set of interpolating functions, $f_{d w}(\phi)=\left(1-2 \phi^{2}+\phi^{4}\right) / 4, \hat{g}(\phi)=\phi-(2 / 3) \phi^{3}+\phi^{5} / 5, h(\phi)$ $=\phi, q_{i}(\phi)=\left\{q_{i}(+1)+q_{i}(-1)+\left[q_{i}(+1)-q_{i}(-1)\right] \phi\right\} / 2$, and $a_{i}(\phi)=1 /(2 \sqrt{2})$ satisfies all the requirements, as detailed for the case of the binary alloy in Ref. [12].

In the case of arbitrary diffusivities, it is difficult to fulfill all the requirements, Eqs. (4.16)-(4.20), simultaneously. Because the antitrapping current is proportional to $\partial_{t} \phi$ and thus $v_{n}$, the contribution of this current vanishes when the interface does not move $\left(v_{n}=0\right)$. The requirements given by Eqs. (4.16) and (4.19) disappear in the case of $v_{n}=0$, since the magnitudes of the interface correction terms associated with them are proportional to $v_{n}$. However, the correction terms associated with Eqs. (4.17), (4.18), and (4.20) are not directly related to $v_{n}$ and these requirements need to be satisfied for $v_{n}=0$ without help of the antitrapping current. This is not a trivial task. Therefore, the antitrapping current proportional to $\partial_{t} \phi$ does not essentially provide a complete solution for the problem in two-sided case as pointed out in Ref. [37]. One of the strategies for overcoming this difficulty is to introduce an additional degree of freedom in choosing the interpolating functions. An attempt was made regarding this point in a recent work [48], where the diffusion mobility $\hat{M}_{c, i}(\phi)$ [or $\left.D_{i}(\phi)\right]$ was defined as a tensor quantity. The essential idea in this tensorial mobility approach is that the interpolating functions for the mobility tensor can be separately defined for normal and parallel components of the diffusion flux in the interface. This relaxes the restrictions in the model. Yet, this approach was developed for a static interface and it has not been extended to describe the moving interface. Our focus is rather the moving interface $\left(v_{n} \neq 0\right)$ as in most of works on microstructural evolution. In this study, we employ the approach put forward for isothermal solidification in a binary alloy [36], which is formally valid as long as $v_{n}$ is finite. The asymptotic analysis shows that the magnitudes of the interface effects associated with Eqs. (4.17) and (4.18) are proportional to the constant $A_{i, 1}(s)$, which depends on the diffusion flux at the interface. By noting this fact, we define a quantity $\chi_{i}$ as follows:

$$
\left.\partial_{r} U_{i, 0}\right|^{-}=-\hat{v}_{n, i} \chi_{i} .
$$

Using $\chi_{i}, a_{i}(\phi)$ is defined for the nonisothermal process in multicomponent alloys as

$$
a_{i}(\phi)=\frac{[h(\phi)-1]\left[q_{i}(-1)-q_{i}(\phi)\right]-2 \chi_{i}\left[q_{i}(+1)-q_{i}(\phi)\right]\left[q_{i}(-1)-q_{i}(\phi)\right]}{2 q_{i}(-1) \partial_{\eta} \phi} .
$$

This approach makes all the correction terms related to Eqs. (4.16)-(4.20) vanish. For the sake of concreteness, we define the interpolating functions as $f_{d w}(\phi)=\left(1-2 \phi^{2}+\right.$ $\left.\phi^{4}\right) / 4, h(\phi)=\phi$, and $q_{i}(\phi)=\left\{q_{i}(+1)+q_{i}(-1)+\left[q_{i}(+1)\right.\right.$ $\left.\left.-q_{i}(-1)\right] \phi\right\} / 2$. Then, $a_{i}(\phi)$ is given by

$$
a_{i}(\phi)=\frac{1}{2 \sqrt{2}}\left[1-\frac{q_{i}(+1)}{q_{i}(-1)}\right] \psi_{i},
$$

with $\psi_{i}=1+\chi_{i}\left[q_{i}(+1)-q_{i}(-1)\right] . u_{i, 1}$ in Eq. (4.15) is now rewritten as

$$
u_{i, 1}=q_{i}(-1)^{-1} \frac{1}{2} \hat{v}_{n, i} \psi_{i} \int_{0}^{\eta}(\phi-1) d \xi-\hat{v}_{n, i} \chi_{i} \eta+A_{i, 2}^{\prime}(s) .
$$

By applying the matching condition to the above equation, one finds $\left.U_{i, 1}\right|^{+}=\left.U_{i, 1}\right|^{-}=\bar{U}_{i, 1}$, viz., no jumps of the diffusion fields at the interface. By substituting Eq. (4.24) into $\phi$ equation at $\varepsilon^{2}$ order, the Gibbs-Thomson relation is obtained without any correction term as follows:

$$
\sum_{i}^{N} M_{i}\left(\bar{U}_{i, 0}+\varepsilon \bar{U}_{i, 1}\right)=-\tilde{\kappa}-\tilde{\beta} v_{n},
$$

where

$$
\tilde{\beta}=\hat{\alpha}-\varepsilon a_{1} a_{2} \sum_{i} \frac{\left[1+\left(1-k_{i}\right) \bar{U}_{i, 0}\right] M_{i}}{q_{i}(-1)} \psi_{i} .
$$

The numerical constants $a_{1}$ and $a_{2}$ are given by $a_{1}=0.8839$ and $a_{2}=0.6267$, respectively. Moreover, $u_{i}$ equation at $\varepsilon^{0}$ leads to the conservation law at interface,

$$
\begin{aligned}
v_{n}[1 & \left.+\left(1-k_{i}\right)\left(\bar{U}_{i, 0}+\varepsilon \bar{U}_{i, 1}\right)\right] \\
= & q_{i}(+1)\left(\left.\partial_{r} U_{i, 0}\right|^{-}+\left.\varepsilon \partial_{r} U_{i, 1}\right|^{-}\right) \\
& -q_{i}(-1)\left(\left.\partial_{r} U_{i, 0}\right|^{+}+\left.\varepsilon \partial_{r} U_{i, 1}\right|^{+}\right) .
\end{aligned}
$$

Since $h(\phi)$ and $q_{i}(\phi)$ used here satisfy Eqs. (4.19) and (4.20), no excess terms appear in this equation.

As described above, the present model is reduced to the sharp-interface equations (4.8)-(4.10) in the thininterface limit. This model does not involve the anomalous interface effects. Therefore, the model should be able to describe quantitatively nonisothermal solidification in a dilute multicomponent alloy with arbitrary diffusivities. However, care must be taken for the fact that the model involves unknown quantities $\left\{\chi_{i}\right\}$, which are originally output quantities related to the diffusion flux. For the symmetric case of $q_{i}(+1)=$ $q_{i}(-1), a_{i}(\phi)$ is null in Eq. (4.23) and $\Psi_{i}=1$. The dependence on $\chi_{i}$ accordingly disappears in this case. For $q_{i}(+1)=0$, the diffusion flux in the solid vanishes and thereby $\chi_{i}=0$ and 
$\Psi_{i}=1$, which makes the model equivalent to the one-sided model $[11,12]$. In arbitrary diffusivities, however, it is not trivial to assign exact values (or function forms) to $\left\{\chi_{i}\right\}$. It is noted that the antitrapping current terms are proportional to $W$ and its contribution decreases with decrease in $W$. Therefore, the result of numerical simulations should be converged to a unique solution as $W$ decreases regardless of values of $\left\{\chi_{i}\right\}$. In this respect, $\left\{\chi_{i}\right\}$ can be regarded as quantities controlling convergence behavior of the solution in this model. In fact, as demonstrated for the isothermal free dendritic growth in a binary alloy [36], the convergence of results with respect to $W$ depends on the value of $\chi_{i}$ and, however, the results are converged to a unique solution regardless of $\chi_{i}$ as $W$ decreases. In the multicomponent case, we have to determine $\chi_{i}$ for each diffusion field. Hence, one may apprehend that the determination of appropriate values of $\left\{\chi_{i}\right\}$ requires a large number of convergence tests. As demonstrated in Sec. V, however, the choice of $\chi_{i}=0$, which is in a strict sense validated for the case of vanishing diffusion current in the solid, yields sufficiently reasonable performance.

\section{Phase-field mobility}

Our focus in this study is the dendritic growth in alloy systems with vanishing kinetic coefficient $(\beta=0)$. Equation (4.26) is rewritten as

$$
\beta=\frac{a_{1} \tau}{\lambda W}\left\{1-a_{2} \frac{\lambda W^{2}}{\tau} \sum_{i} \frac{\left[1+\left(1-k_{i}\right) \bar{U}_{i, 0}\right] M_{i}}{D_{i}(-1)} \psi_{i}\right\} .
$$

It is noted that $\beta$ depends on $\bar{U}_{i, 0}$. For $k_{i}=1$, the dependence on $\bar{U}_{i, 0}$ disappears and $\beta=0$ is achieved by choosing $\tau=$ $a_{2} \lambda W^{2} \Sigma_{i} M_{i} \Psi_{i} / D_{i}$. For $k_{i} \neq 1, \beta$ can be made to vanish by setting $\tau=a_{2} \lambda W^{2} \Sigma_{i}\left[1+\left(1-k_{i}\right) \bar{U}_{i, 0}\right] M_{i} \Psi_{i} / D_{i}$. However, $\left\{\bar{U}_{i, 0}\right\}$ are not a priori known. These values are mutually dependent and are related to the local shape and velocity of the interface. Therefore, the difficulty arises in making $\beta$ completely vanish. Following the previous works on binary systems $[12,14]$, in this study, $\tau$ is made $\left\{u_{i}\right\}$-dependent to tackle this problem,

$$
\tau=\tau_{0} \sum_{i} \frac{\left[1+\left(1-k_{i}\right) u_{i}\right] M_{i}}{q_{i}(-1)} \psi_{i}
$$

where $\tau_{0}=a_{2} \lambda W^{2} / D_{l, \text { ref. }}$. This is identical to Eq. (3.11). This modification does not change the asymptotic analysis for $u_{i}$ fields. By repeating the analysis for $\phi$ equation, one finds that $\hat{\alpha}$ in Eq. (4.11) is replaced by $\hat{\alpha}_{0} \sum\left[1+\left(1-k_{i}\right) \bar{U}_{i, 0}\right] M_{i} \psi_{i} / q_{i}(-1)$ where $\hat{\alpha}_{0}=$ $D_{l, \text { ref }} \tau_{0} / W^{2}$. The relation for $\bar{U}_{i, 1}$ is obtained from $\phi$ equation at $\varepsilon^{2}$ order and $\tilde{\beta}$ in Eq. (4.25) is now given by

$$
\begin{aligned}
\tilde{\beta}= & a_{1} a_{2} \varepsilon^{2} \sum_{i} \frac{\left(1-k_{i}\right) M_{i} \psi_{i}}{q_{i}(-1)} \\
& \times\left\{\bar{U}_{i, 1}-v_{n} a_{3} \frac{\left[1+\left(1-k_{i}\right) \bar{U}_{i, 0}\right] \psi_{i}}{q_{i}(-1)}\right\},
\end{aligned}
$$

where $a_{3}$ is a numerical constant. Therefore, as long as this quantity proportional to $\varepsilon^{2}$ is much smaller than unity, the kinetic effect in the Gibbs-Thomson relation is negligible.

\section{Dependence of diffusivities on diffusion fields}

The solutal and thermal diffusivities in reality depend on the temperature and concentrations. We consider the dependence of the diffusivity on $\left\{u_{i}\right\} . D_{i}(\phi)$ in Eq. (4.4) is redefined as $D_{i}\left(\phi,\left\{u_{i}\right\}\right)$, where $D_{i}\left(+1,\left\{u_{i}\right\}\right)=k_{i} D_{s, i}\left(\left\{u_{i}\right\}\right)$ and $D_{i}\left(-1,\left\{u_{i}\right\}\right)=D_{l, i}\left(\left\{u_{i}\right\}\right) \cdot q_{i}(\phi)$ in Eq. (4.7) is accordingly replaced by $q_{i}\left(\phi,\left\{u_{i}\right\}\right)=D_{i}\left(\phi,\left\{u_{i}\right\}\right) / D_{l, \text { ref }}$ with a constant $D_{l \text {,ref. }}$ For simplicity, although not necessary, our discussion is given for the case of $f_{d w}(\phi)=\left(1-2 \phi^{2}+\phi^{4}\right) / 4, h(\phi)=\phi$ and $q_{i}\left(\phi,\left\{u_{i}\right\}\right)=\left\{q_{i}\left(+1,\left\{u_{i}\right\}\right)+q_{i}\left(-1,\left\{u_{i}\right\}\right)+\left[q_{i}\left(+1,\left\{u_{i}\right\}\right)\right.\right.$ $\left.\left.-q_{i}\left(-1,\left\{u_{i}\right\}\right)\right] \phi\right\} / 2$. The interpolating function in the antitrapping current term $a_{i}$ is redefined to depend on $\left\{u_{i}\right\}$ as follows:

$$
a_{i}\left(\phi,\left\{u_{i}\right\}\right)=\frac{1}{2 \sqrt{2}}\left[1-\frac{q_{i}\left(+1,\left\{u_{i}\right\}\right)}{q_{i}\left(-1,\left\{u_{i}\right\}\right)}\right] \psi_{i}\left(\left\{u_{i}\right\}\right),
$$

where $\psi_{i}\left(\left\{u_{i}\right\}\right)=1+\chi_{i}\left[q_{i}\left(+1,\left\{u_{i}\right\}\right)-q_{i}\left(-1,\left\{u_{i}\right\}\right)\right]$. These modifications do not alter the solvability conditions for $\phi$; the asymptotic analysis for $\phi$ equation is identical to that explained in Sec. IV B. $q_{i}\left(\phi_{0}\right)$ in Eqs. (4.12)-(4.20) is now replaced with $q_{i}\left(\phi_{0},\left\{\bar{U}_{i, 0}\right\}\right)$. All the constraints described by Eqs. (4.16)-(4.20) are simultaneously satisfied. The difference appears in $u_{i}$ equation at $\varepsilon^{0}$ order. The resulting expression for conservation condition of outer fields at the interface contains new extra term given by $\varepsilon \sum_{j}\left(\partial_{s} U_{i, 0} \partial_{s} U_{j, 0}\right)\left(Q_{i j}^{+}-Q_{i j}^{-}\right)$with

$Q_{i j}^{ \pm}=\int_{0}^{ \pm \infty}\left[\left.\frac{\partial q_{i}\left(\phi_{0},\left\{u_{i}\right\}\right)}{\partial u_{j}}\right|_{*}-\left.\frac{\partial q_{i}\left(\mp 1,\left\{u_{i}\right\}\right)}{\partial u_{j}}\right|_{*}\right] d \eta$

The asterisk indicates that the derivative is evaluated at $\left\{u_{i}\right\}=$ $\left\{\bar{U}_{i, 0}\right\}$. In order to purge this extra term, $Q_{i j}^{+}=Q_{i j}^{-}$must be satisfied. This is the additional constraint. However, the form of $q_{i}\left(\phi,\left\{u_{i}\right\}\right)$ chosen here actually satisfies the constraint, $Q_{i j}^{+}=$ $Q_{i j}^{-}$without any modification. As a result, one obtains the conservation condition for the outer fields to first order as follows:

$$
\begin{aligned}
v_{n}[1 & \left.+\left(1-k_{i}\right)\left(\bar{U}_{i, 0}+\varepsilon \bar{U}_{i, 1}\right)\right] \\
= & {\left.\left[q_{i}\left(+1,\left\{\bar{U}_{i, 0}\right\}\right)+\left.\varepsilon \sum_{j} \frac{\partial q_{i}\left(+1,\left\{u_{i}\right\}\right)}{\partial u_{j}}\right|_{*} \bar{U}_{j, 1}\right] \partial_{r} U_{i, 0}\right|^{-} } \\
& +\left.\varepsilon q_{i}\left(+1,\left\{\bar{U}_{i, 0}\right\}\right) \partial_{r} U_{i, 1}\right|^{-} \\
& -\left[q_{i}\left(-1,\left\{\bar{U}_{i, 0}\right\}\right)+\left.\varepsilon \sum_{j} \frac{\partial q_{i}\left(+1,\left\{u_{i}\right\}\right)}{\partial u_{j}}\right|_{*} \bar{U}_{j, 1}\right] \\
& \times\left.\partial_{r} U_{i, 0}\right|^{+}-\left.\varepsilon q_{i}\left(-1,\left\{\bar{U}_{i, 0}\right\}\right) \partial_{r} U_{i, 1}\right|^{+} .
\end{aligned}
$$

Therefore, without any modification, $\left\{u_{i}\right\}$ dependence on the diffusivity can be taken into account in this model. This is an important feature for description and prediction of solidification structures in real alloy systems. However, for the sake of simplicity, we employ constant diffusivities for $D_{s, i}$ and $D_{l, i}$ in the numerical tests of the model, the purpose of which is to check the convergence behavior of the simulation outcome in model systems. 


\section{E. Anisotropy}

The crystalline anisotropy is included in this model by letting $W$ and $\tau$ in Eq. (4.3) depend on $\vec{n}$. As in the standard manner [9], $W(\vec{n})$ and $\tau(\vec{n})$ are given for fourfold symmetry by

$$
\begin{gathered}
W(\vec{n})=W_{0} a_{s}(\vec{n}) \\
=W_{0}\left(1-3 \varepsilon_{4}\right)\left[1+\frac{4 \varepsilon_{4}}{1-3 \varepsilon_{4}} \frac{\left(\partial_{x} \phi\right)^{4}+\left(\partial_{y} \phi\right)^{4}}{|\nabla \phi|^{4}}\right], \\
\tau(\vec{n})=\tau_{a} a_{s}(\vec{n})^{2},
\end{gathered}
$$

where $\varepsilon_{4}$ is the parameter characterizing the anisotropy strength and $\tau_{a}$ is a constant. When $\beta=0$ is considered, $\tau_{a}$ is replaced with $\left\{u_{i}\right\}$-dependent form given by Eq. (4.29). Then, $\phi$ equation (4.3) is rewritten as

$$
\begin{aligned}
\tau_{0} \xi( & \left(\left\{u_{i}\right\}\right) a_{s}(\vec{n})^{2} \partial_{t} \phi \\
= & W_{0}^{2} \nabla \cdot\left[a_{s}(\vec{n})^{2} \nabla \phi\right]+W_{0}^{2} \partial_{x}\left[|\nabla \phi|^{2} a_{s}(\vec{n}) \frac{\partial a_{s}(\vec{n})}{\partial\left(\partial_{x} \phi\right)}\right] \\
& +W_{0}^{2} \partial_{y}\left[|\nabla \phi|^{2} a_{s}(\vec{n}) \frac{\partial a_{s}(\vec{n})}{\partial\left(\partial_{y} \phi\right)}\right]-f_{d w}^{\prime}-\hat{g}^{\prime}(\phi) \Delta f_{\text {driv }},
\end{aligned}
$$

where $f_{d w}^{\prime}=-\phi+\phi^{3}, \hat{g}^{\prime}(\phi)=\left(1-\phi^{2}\right)^{2}$ and

$$
\begin{gathered}
\xi\left(\left\{u_{i}\right\}\right)=\sum_{i=1}^{N} \frac{\left[1+\left(1-k_{i}\right) u_{i}\right] M_{i} \psi_{i}}{q_{i}(-1)}, \\
\Delta f_{\text {driv }}=\lambda\left(\sum_{i=1}^{N} M_{i} u_{i}\right) .
\end{gathered}
$$

It is noted that the antitrapping current terms contain the interface thickness $W$. In the numerical tests described in the next section, we focus on the small value of $\varepsilon_{4}$, as is the case for most metallic alloys and the anisotropy of $W$ in the antitrapping current terms is omitted for simplicity. The morphology of the microstructure is sensitive to the anisotropy of capillary length, which is 15 times larger than the one of $W$ for fourfold symmetry and only a small error will appear by this simplification [12]. However, this simplification cannot be validated for some alloys where the anisotropic strength, depending on alloy composition, becomes relatively large [49].

\section{NUMERICAL TESTS}

\section{A. Computational details}

In order to check the performance of the model, we have simulated free dendritic growth in binary and ternary alloys. Equations (4.36), (4.4), and (4.5) were discretized in a two-dimensional system based on standard second-order finite difference formulas with a square grid spacing $\Delta x$. The time evolutions of $\phi$ and $u_{i}$ fields were solved using a simple first-order Euler scheme.

We employed a computational box of rectangular shape. The simulations start from a small quarter disk of solid at one corner of the box $(x=0$ and $y=0)$ in spatially uniform undercooling or supersaturation described by $\left\{u_{i, \text { initial }}\right\}$. The size of the disk was $20 d_{0}$ in all the simulations. To reduce the computational time, the simulations were conducted only in the quadrant $x>0$ and $y>0$. A symmetry boundary condition was applied on the bottom and left sides. A fixed far-field condition, viz., condition of $\phi=-1$ and $\left\{u_{i}\right\}=\left\{u_{i \text {,initial }}\right\}$ was used on the top and right sides. We focused on the growth of dendrite along the $y$ direction. Care has to be taken that the diffusion fields along the $y$ direction do not reach the end of the computational box. In order to describe free dendritic growth into an infinite melt, the computational box needs to be large enough that the dendrite can reach the steady growth regime before the diffusion boundary layers reach the far end of the system. This is computationally demanding especially in the case of nonisothermal solidification because the Lewis number Le, the ratio of thermal diffusivity to solutal diffusivity, is generally several orders of magnitude larger than unity and hence the thermal diffusion length is much larger than the solutal diffusion length. In the previous studies, a nonlinear preconditioning method for the $\phi$ equation, which enables the computation with large mesh size [18], or an implicit, adaptive finite-difference method [24] was utilized to reduce the computational time. In this study, the simulations were accelerated by using a graphics processing unit (GPU). The GPU implementation of phase-field simulation for a binary alloy was reported in Ref. [50]. A program code was written by computer unified device architecture (CUDA) FORTRAN and executed on a TESLA C2075 GPU in this study. In addition, a moving frame method was used. The computational box was moved with a certain velocity in the $-y$ direction, which, within a reasonable computational time, allows one to track the dendrite tip growing along $y$ direction until the steady-state is reached. The frame was moved so that the diffusion fields at the top edge of the $y$ axis do not increase by more than $2 \times 10^{-6} \%$ from their initial values and this avoids the effect of any wall interaction.

In all the simulations, we employed $W_{0}=1$ and $\tau_{0}=$ 1 and hence, $D_{l, \text { ref }}=a_{2} \lambda$ according to the relation $\tau_{0}=$ $a_{2} \lambda W_{0}^{2} / D_{l, \text { ref }}$ for $\beta=0$. The time step was set to $\Delta t=$ $\Delta x^{2} /\left(5 D_{\text {max }}\right)$ with a maximum diffusivity in the system, $D_{\text {max }}$. The anisotropy strength $\varepsilon_{4}$ was set to $\varepsilon_{4}=0.02$ in all the simulations. The convergence tests were performed for the range of $W_{0} / d_{0}$ from 1.13 to 10 , which corresponds to the range of $\lambda$ from 1.0 to $8.84\left(\lambda=a_{1} W_{0} / d_{0}\right)$.

\section{B. Binary system}

We first focus on nonisothermal solidification in a binary alloy. The thermal diffusivity in solid is assumed to be equal to that in the liquid, $D_{s, T}=D_{l, T}$ (symmetric heat transport). Then, Eqs. (4.37) and (4.38) are explicitly given by

$$
\begin{gathered}
\xi(u)=\mathrm{Le}^{-1}+[1+(1-k) u] M \psi, \\
\Delta f_{\text {driv }}=\lambda(\theta+M u),
\end{gathered}
$$

where Le is the Lewis number given by $L e=D_{l, T} / D_{l}$ with the solutal diffusivity in the liquid $D_{l}\left(=D_{l, \text { ref }}\right)$ and $M$ is given by $M=d_{0} / d_{s}$ with the thermal capillary length $d_{0}$ and the chemical capillary length $d_{s}$. The diffusion equations are 


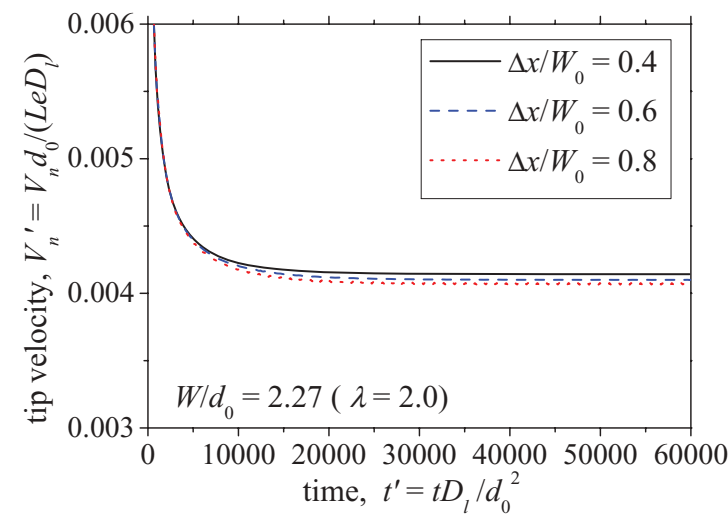

FIG. 1. (Color online) Temporal variation of tip velocity during two-dimensional free dendritic growth in a binary alloy, calculated for $\theta_{\text {initial }}=-0.55, L e=40, M=0.07, k=0.15, \varepsilon_{4}=0.02, D_{s}=$ 0.0 , and $W_{0} / d_{0}=2.27(\lambda=2.0)$.

written as

$$
\begin{aligned}
\frac{1}{2}[1 & +k-(1-k) \phi] \partial_{t} u \\
= & \nabla\left\{\frac{1}{2}\left[k D_{s}+D_{l}+\left(k D_{s}-D_{l}\right) \phi\right] \nabla u\right. \\
& \left.+a_{A T}\left(1-\frac{k D_{s}}{D_{l}}\right) \psi W_{0}[1+(1-k) u] \partial_{t} \phi \frac{\nabla \phi}{|\nabla \phi|}\right\}
\end{aligned}
$$

(a)

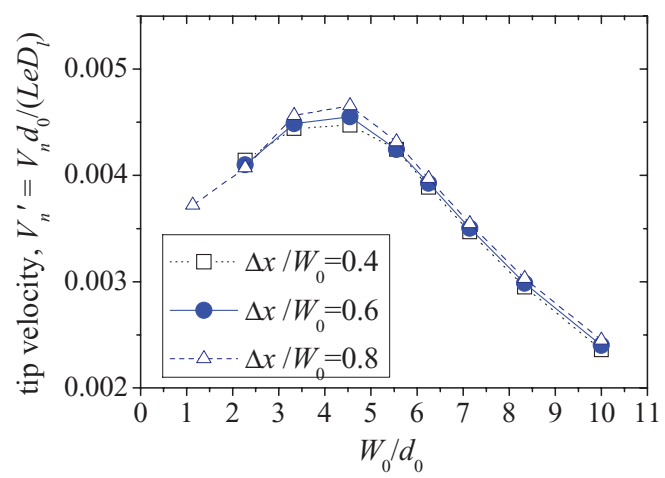

(c)

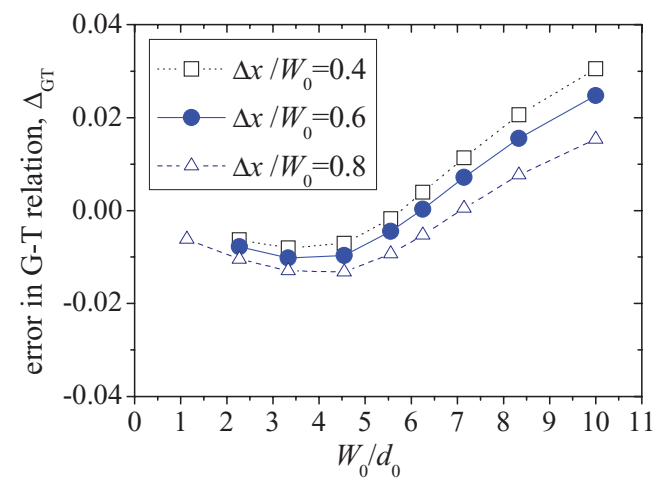

$$
\begin{aligned}
+\frac{1}{2}[1+(1-k) u] \partial_{t} \phi & \\
\partial_{t} \theta & =D_{l} \operatorname{Le} \nabla^{2} \theta+\frac{\partial_{t} \phi}{2},
\end{aligned}
$$

where $k$ is the equilibrium partition coefficient, $D_{s}$ is solutal diffusivity in the solid and $a_{A T}$ is given by $a_{A T}=1 /(2 \sqrt{2})$. It is noted that $\Psi$ is given by $\Psi=1+\chi\left(k D_{s} / D_{l}-1\right)$ with $\chi$ the parameter related to the diffusion flux in the solid. The model developed in Ref. [14] is reproduced by putting $D_{s}=$ $0, \chi=0$, and $\Psi=1$ in these equations.

Nonisothermal dendritic growth into the undercooled melt was investigated using the model for symmetric thermal diffusion and one-sided solutal diffusion in the previous studies $[14,18,24,27,32]$. Large variations of the steady-state velocity and radius of the growing dendrite tip were observed for different values of $\Delta x / W_{0}[18]$. As $\Delta x / W_{0}$ increases from 0.3 to 0.8 , the tip velocity $V_{n} d_{0} /\left(\operatorname{Le} D_{l}\right)$ decreases from about $4.1 \times 10^{-3}$ to about $3.6 \times 10^{-3}$ (error of $\sim 12 \%$ ) and the tip radius $\rho / d_{0}$ increases from about 27 to about 32 (error of $\sim 14 \%$ ). Hence, it was shown that the small value of $\Delta x$ $\leqslant 0.3$ has to be used to obtain the accurate solution. In their work [18], this problem was overcome by using the nonlinear preconditioning method for the $\phi$ equation which allows accurate simulations for larger value of $\Delta x$ [51].

The dependence of the result on $\Delta x$ is reinvestigated in this study. Figure 1 shows the temporal change of tip velocity

(b)

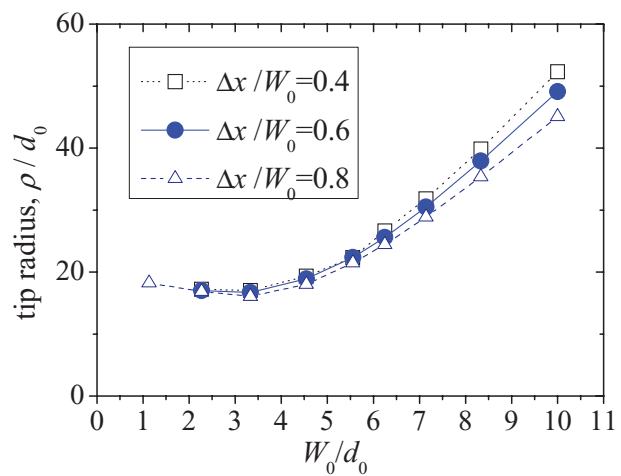

(d)

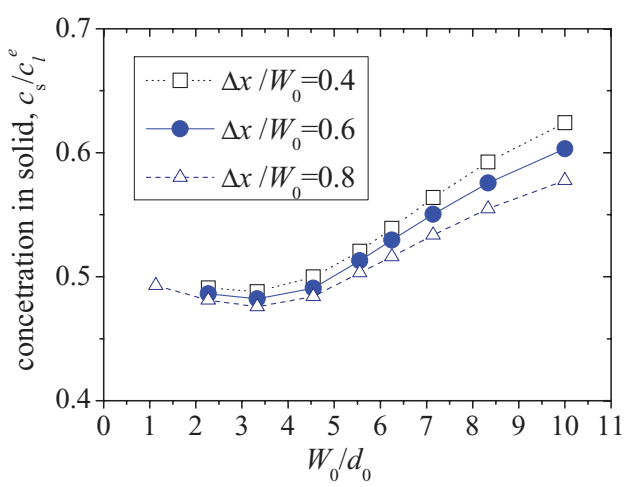

FIG. 2. (Color online) Convergence behavior of (a) tip velocity, (b) tip radius, (c) error in Gibbs-Thomson relation given by Eq. (5.5), and (d) concentration in solid at the tip, with respect to $W_{0} / d_{0}$. These are steady-state values for two-dimensional free dendritic growth in a binary alloy calculated for $\theta_{\text {initial }}=-0.55, L e=40, M=0.07, k=0.15, \varepsilon_{4}=0.02$, and $D_{s}=0.0$. 
(a)

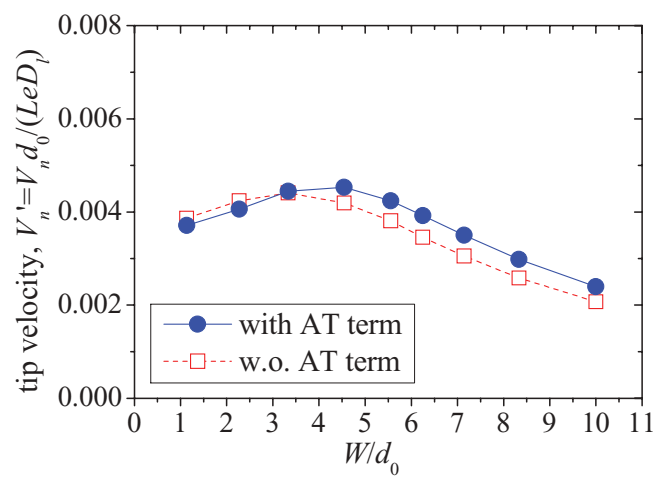

(c)

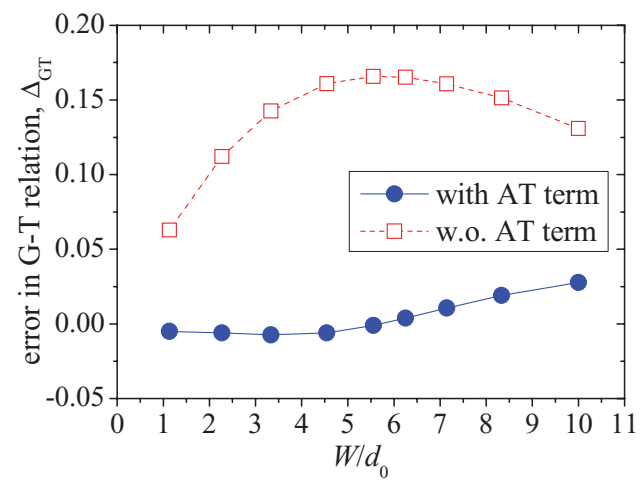

(b)

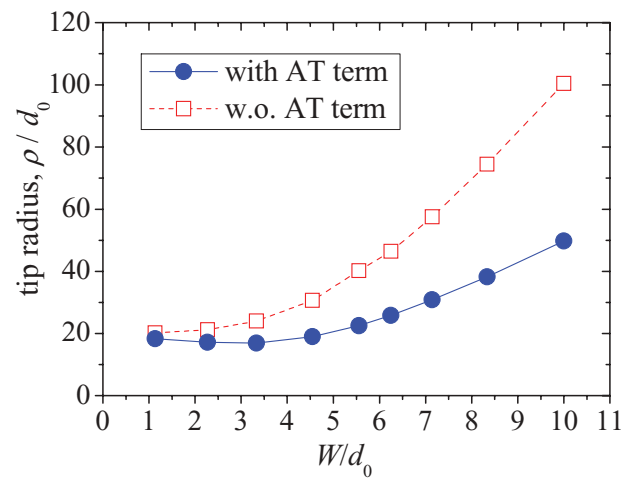

(d)

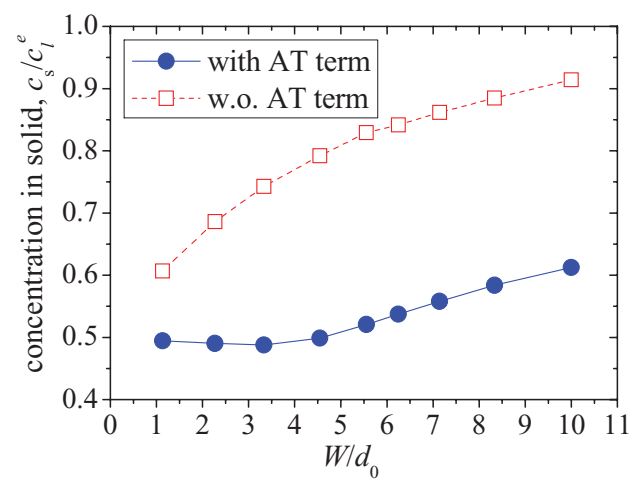

FIG. 3. (Color online) Convergence behavior of (a) tip velocity, (b) tip radius, (c) error in Gibbs-Thomson relation, and (d) concentration in solid at the tip, with respect to $W_{0} / d_{0}$. These are the steady-state values for two-dimensional free dendritic growth in a binary alloy calculated for $\theta_{\text {initial }}=-0.55, L e=40, M=0.07, k=0.15, \varepsilon_{4}=0.02$, and $D_{s} / D_{l}=0.1$.

calculated for $\Delta x / W_{0}=0.4,0.6$, and 0.8 . The simulations are for $\theta_{\text {initial }}=-0.55, u_{\text {initial }}=0.0$, Le $=40, M=0.07, k=$ $0.15, \varepsilon=0.02, D_{s}=0.0(\chi=0.0, \Psi=1.0), W_{0} / d_{0}=2.27$ $(\lambda=2.0)$. These parameters should be the same as those used for Fig. 3 of Ref. [18]. From preliminary calculations, we found that the system size has to be larger than $960 d_{0}$ ( $x$ axis) $\times 1920 d_{0}$ ( $y$ axis) to avoid the effect of walls of the computational box. All the calculations given in Sec. V B were conducted in the system larger than this size. In Fig. 1, the dendritic growth reaches the steady-state regime in each case. The steady-state values were calculated by averaging the velocity over the time period of $t D_{l} / d_{0}^{2}=5.5 \times 10^{4}$ $-6.0 \times 10^{4}$. The steady-state velocities were calculated to be $4.14 \times 10^{-3}$ for $\Delta x / W_{0}=0.4,4.10 \times 10^{-3}$ for $\Delta x / W_{0}=$ 0.6 and $4.07 \times 10^{-3}$ for $\Delta x / W_{0}=0.8$. Hence, contrary to the results in Ref. [18], we found very small error of less than $1.7 \%$ in this range of $\Delta x / W_{0}$ without the nonlinear preconditioning technique. It is noted that there are differences in the numerical conditions and technique used in the present and the previous studies [18]. For example, in Ref. [18], the effect of walls of computational box was considered negligible as long as the temperature at the top edge of the growing direction does not increase by more than $0.5 \%$, which is larger than $2.0 \times 10^{-6} \%$ used in this study. In addition, a mesh adaptation method for space was used in their work to deal with relatively large computational box. Although not exactly clear, the different convergence with respect to $\Delta x$ might be related to the different numerical conditions and technique. As shown below, on the other hand, our results of the convergence behavior with respect to $W_{0} / d_{0}$ (or $\lambda$ ) are very similar to their results obtained using the preconditioning technique, which should be the most precise in their work [18].

Figure 2(a) displays the convergence of the steady-state velocity of dendrite tip with respect to $W_{0} / d_{0}$ calculated for three different values of $\Delta x / W_{0}$. As for $W_{0} / d_{0}=1.13$ $(\lambda=1.0)$, the simulation was performed only for $\Delta x / W_{0}=$ 0.8 , since extremely large computational time was required for $\Delta x / W_{0}=0.4$ and 0.6 . In all the cases, the velocity first increases and then decreases with increase in $W_{0} / d_{0}$. This result can be directly compared to Fig. 5 of Ref. [18] where the horizontal axis is $\lambda\left(=a_{1} W_{0} / d_{0}\right)$. One can notice that the convergence behavior in this work is quite consistent with that calculated based on the preconditioning method in the previous study [18]. On the other hand, importantly, our simulations show that the computational results are not sensitive to the choice of $\Delta x / W_{0}$ for each value of $W_{0} / d_{0}$.

The calculated tip radius is shown in Fig. 2(b). The tip radius was calculated by fitting $\phi=0$ contour at the dendrite tip into a parabola. In Fig. 2(b), the convergence seems to break down for $W_{0} / d_{0} \sim 4$. This behavior is fairly similar to that shown in Fig. 5 of Ref. [18], while the values are slightly different due to a different estimation way of the radius. Figure 2(c) 
(a)

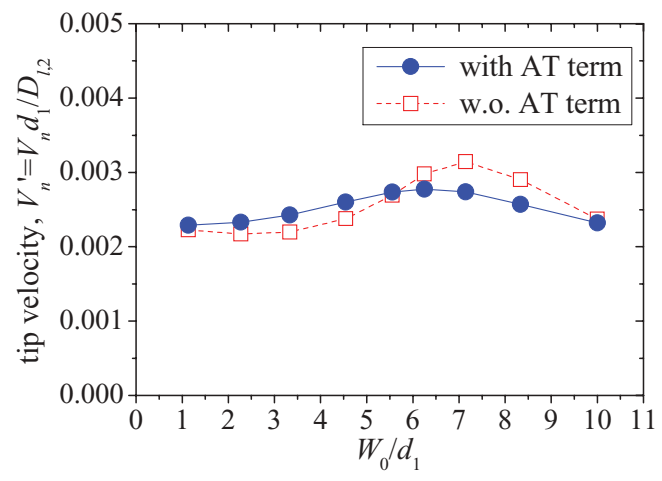

(c)

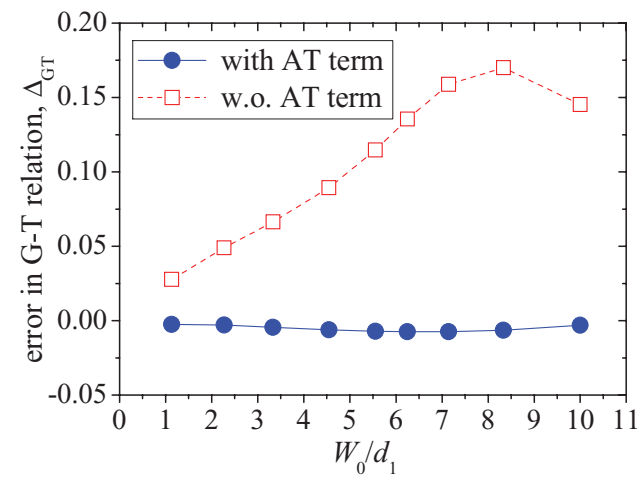

(b)

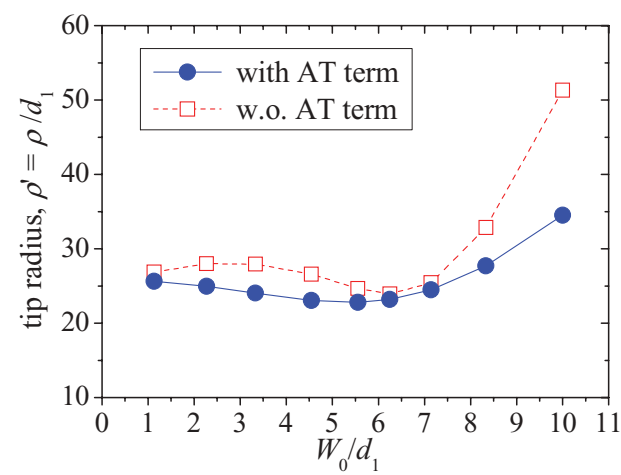

(d)

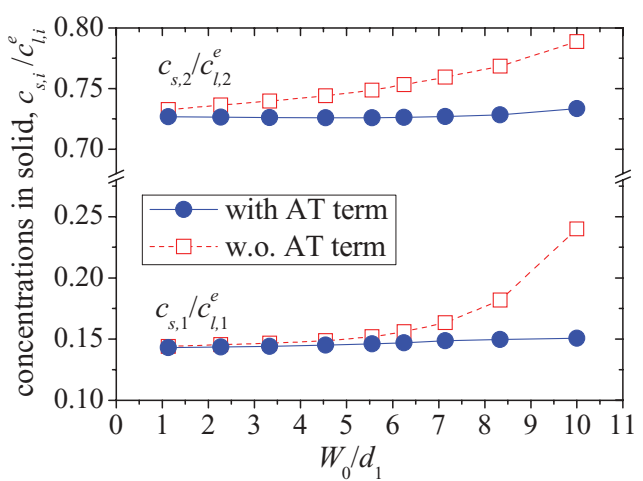

FIG. 4. (Color online) Convergence behavior of (a) tip velocity, (b) tip radius, (c) error in Gibbs-Thomson relation, and (d) concentrations in solid at the tip, with respect to $W_{0} / d_{1}$. These are steady-state values for isothermal dendritic growth in a ternary alloy calculated for $u_{1, \text { initial }}=$ $u_{1, \text { initial }}=-0.4, M_{2}=0.9, k_{1}=0.16, k_{2}=0.7, D_{l, 1} / D_{l, 2}=5.0, D_{s, 1} / D_{l, 2}=1.5, D_{s, 2} / D_{l, 2}=0.006$, and $\varepsilon_{4}=0.02$.

shows the error in the Gibbs-Thomson relation, $\Delta_{\mathrm{GT}}$. The Gibbs-Thomson relation with the anisotropy is written as $\theta^{*}+M u^{*}=-d_{0}(\vec{n}) \kappa$, where $\theta^{*}$ and $u^{*}$ are the values at the interface. $d_{0}(\vec{n})$ is replaced with $d_{0}\left(1-15 \varepsilon_{4}\right)$ when applied in the direction of vertical dendrite growth axis. Then, the quantity

$$
\Delta_{\mathrm{GT}}=\theta^{*}+M u^{*}+d_{0}\left(1-15 \varepsilon_{4}\right) / \rho
$$

represents the error in the Gibbs-Thomson relation. When the Gibbs-Thomson relation is exactly satisfied, $\Delta_{\mathrm{GT}}$ should be null. $\theta^{*}$ and $u^{*}$ were estimated at a region of $\phi=0.95-0.97$ as is similar to that in Ref. [14]. $\Delta_{\mathrm{GT}}$ is very small when $W_{0} / d_{0}$ is less than 4 and then it gradually increases with increase in $W_{0} / d_{0}$. The concentration in the solid calculated from $u^{*}$ is shown in Fig. 2(d) and the result is well converged for $W_{0} / d_{0}$ $\sim 4$ (or $W_{0} / \rho \sim 0.2$ ).

From Fig. 2, it is evident that the convergence behavior is not sensitive to the choice of $\Delta x$. The simulation with $\Delta x / W_{0}=0.6$ yields reasonable accuracy. Hence, we employed $\Delta x / W_{0}=0.6$ in all the simulations discussed below. Only for $W_{0} / d_{0}=1.13(\lambda=1), \Delta x / W_{0}=0.8$ was employed to save the computational time.

Now we focus on nonisothermal free dendritic growth in a binary alloy with a finite solutal diffusivity in the solid. The input parameters and numerical conditions are the same as those used above except that $D_{s} / D_{l}$ is set to 0.1 . It is noted that the parameter controlling the convergence behavior $\chi$ has to be given in this case. We assumed $\chi=0.0$ and thus $\Psi_{s}=1.0$. Although $\chi=0.0$ is validated for the case of vanishing diffusion current in the solid, this choice yielded excellent convergence behavior for the isothermal dendritic growth in a binary alloy [36]. Figure 3 shows the calculated convergence behavior. The circle symbols indicate the results obtained by this model, while the square symbols are those by the model without the antitrapping current, viz., the model with $a_{\mathrm{AT}}=0$ in Eq. (5.3). These models are called the model with AT and the model without AT in the following discussions. The behavior of the model with AT are very similar to those of one-sided model shown in Fig. 2. Reasonable convergence behavior of each quantity is observed until $W_{0} / d_{0} \sim$ $4\left(W_{0} / \rho \sim 0.2\right)$ and then the convergence starts to break down as $W_{0} / d_{0}$ increases. The advantage of the model with AT is obvious in comparison with the model without AT. Although only a slightly worse convergence is observed for the velocity in the model without AT, the convergence of tip radius is much worse. Furthermore, the error in the Gibbs-Thomson relation is substantial in the model without AT. Even at $W_{0} / d_{0}=1.13$ $(\lambda=1.0)$, the error amounts to 0.06 , which should be associated with the $\theta^{*}+M u^{*}$ term in Eq. (5.5) because $\rho$ is almost converged. The concentration in solid is not fully converged 
(a)

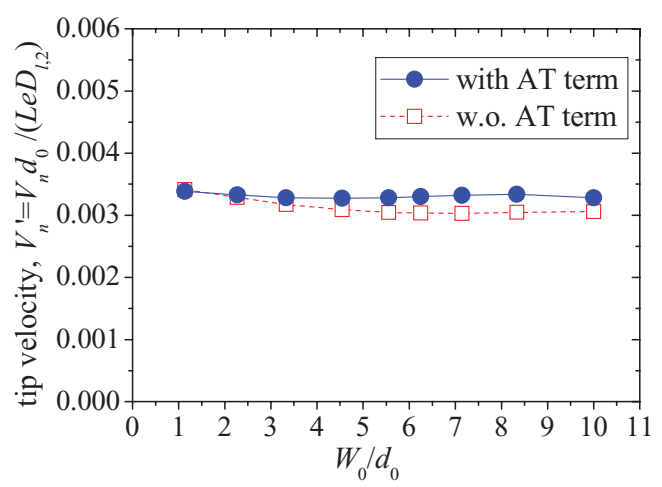

(c)

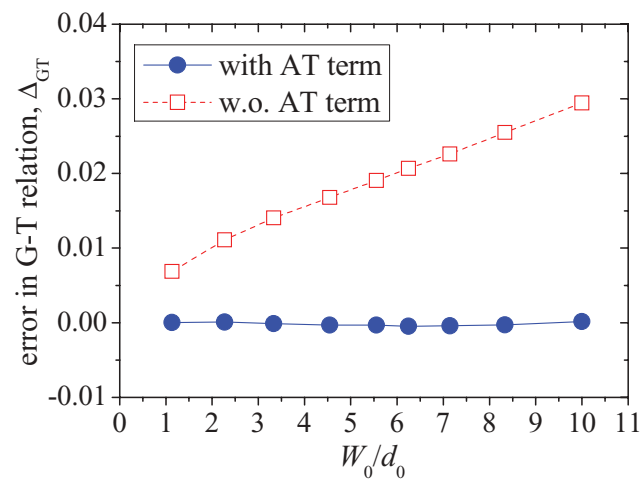

(b)

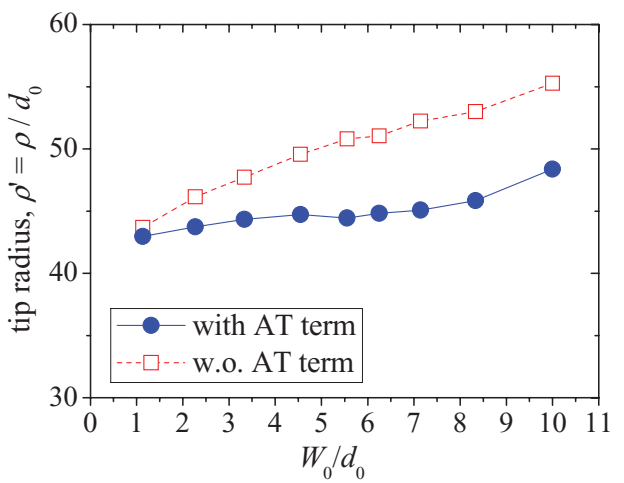

(d)

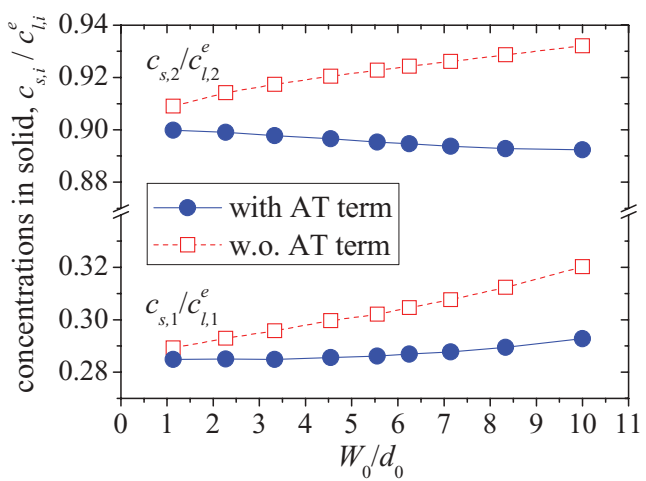

FIG. 5. (Color online) Convergence behavior of (a) tip velocity, (b) tip radius, (c) error in Gibbs-Thomson relation, and (d) concentrations in solid at the tip, with respect to $W_{0} / d_{0}$. These are steady-state values for nonisothermal dendritic growth in a ternary alloy calculated for $\theta_{\text {initial }}=$ $-0.55, u_{1, \text { initial }}=u_{1 \text {,initial }}=0.0, M_{1}=0.07, M_{2}=0.063, k_{1}=0.16, k_{2}=0.7, L e=5.0, D_{l, 1} / D_{l, 2}=5.0, D_{s, 1} / D_{l, 2}=1.5, D_{s, 2} / D_{l, 2}=0.006$, and $\varepsilon_{4}=0.02$.

in the model without AT. The comparison in Fig. 3 strongly indicates the superiority of the present model (the model with AT). Reasonable performance is realized in the present model even with $\chi=0$.

\section{Ternary system}

Our focus is first placed on the isothermal solidification in a ternary alloy. The time evolution equations for two concentrations $u_{1}, u_{2}$ and for $\phi$ were solved in this case. The subscript, $i$, is added to all the quantities in Eq. (5.3) except for $a_{\mathrm{AT}}, \phi$, and $W_{0}$ and the equations were solved for $i=1$ and 2. The time evolution of $\phi$ is described by Eq. (4.36) with $\xi\left(\left\{u_{i}\right\}\right)$ and $\Delta f_{\text {driv }}$ given by

$$
\begin{gathered}
\xi\left(u_{1}, u_{2}\right)=D_{l, \text { ref }}\left\{\frac{\left[1+\left(1-k_{1}\right) u_{1}\right] M_{1} \psi_{1}}{D_{l, 1}}\right. \\
\left.+\frac{\left[1+\left(1-k_{2}\right) u_{2}\right] M_{2} \psi_{2}}{D_{l, 2}}\right\}, \\
\Delta f_{\text {driv }}=\lambda\left(M_{1} u_{1}+M_{2} u_{2}\right) .
\end{gathered}
$$

In the simulations, $D_{l, \text { ref }}$ was set to $D_{l, 2}$ and the spatial scale was measured in unit of chemical capillary length of element $1, d_{1} . M_{1}$ and $\lambda$ are given by $M_{1}=1$ and $\lambda=a_{1} W_{0} / d_{1}$, respectively. Then, $M_{2}=d_{1} / d_{2}$ is the ratio of $d_{1}$ to the chemical capillary length of element $2, d_{2}$.
The simulations were performed for $u_{1 \text {,initial }}=u_{2 \text {,initial }}=$ $-0.4, k_{1}=0.16, k_{2}=0.7, M_{2}=0.9, D_{l, 1} / D_{l, 2}=5.0$, $D_{s, 1} / D_{l, 2}=1.5$, and $D_{s, 2} / D_{l, 2}=0.006$. The relation for the diffusivities used here is close to that in Fe-C-Mn system. $\chi_{1}$ and $\chi_{2}$ were given by $\chi_{1}=\chi_{2}=0$. From preliminary calculations, it was found that the system size has to be larger than $1500 d_{1}$ ( $x$ axis) $\times 3000 d_{1}$ ( $y$ axis) to avoid the effect of walls of the computational box. The convergence behavior is shown in Fig. 4 where the results of the models with and without AT are indicated by circle and square symbols, respectively. $\Delta_{\mathrm{GT}}$ is now given by $\Delta_{\mathrm{GT}}=M_{1} u_{1}^{*}+M_{2} u_{2}^{*}+$ $d_{1}\left(1-15 \varepsilon_{4}\right) / \rho$. The steady-state value of each quantity was obtained by averaging over the time period of $t D_{l, 2} / d_{1}^{2}=$ $5.5 \times 10^{5}-6.0 \times 10^{5}$. Better convergence is obtained by the model with AT. Although the similar convergence for $V_{n}^{\prime}$ and $\rho^{\prime}$ are obtained by both the models in the range of $W_{0} / d_{1} \leqslant$ 6 , a salient difference appears in $\Delta_{\mathrm{GT}}$ even in this range. This indicates that the accuracy of the model should be judged not only by $V_{n}^{\prime}$ and $\rho^{\prime}$ but also by $\Delta_{\mathrm{GT}}$ and/or the concentration profile.

Last we discus nonisothermal dendritic growth in a ternary alloy. As for heat transport, equal thermal diffusivities in the solid and liquid are assumed. The time evolution of $\theta$ given by Eq. (5.4) was solved in addition to $u_{1}$ and $u_{2}$ equations described above. $D_{l, \text { ref }}$ was taken to be $D_{l, 2}$ and the spatial 
scale was normalized by $d_{0}$. $\mathrm{Le}^{-1}$ and $\lambda \theta$ terms are added to Eqs. (5.6) and (5.7), respectively. Here the definitions of the quantities are Le $=D_{l, T} / D_{l, 2}, \lambda=a_{1} W_{0} / d_{0}, M_{1}=d_{0} / d_{1}$, and $M_{2}=d_{0} / d_{2}$. Le was set to small value of 5.0 since a higher value of Le requires a larger system to be treated due to larger thermal diffusion layer and it also requires a smaller time step. The simulations were performed for $\theta_{\text {initial }}=-0.55, u_{1, \text { initial }}=u_{2, \text { initial }}=0, k_{1}=0.16, k_{2}=$ $0.7, M_{1}=0.07 M_{2}=0.063, D_{l, 1} / D_{l, 2}=5.0, D_{s, 1} / D_{l, 2}=$ $1.5, D_{s, 2} / D_{l, 2}=0.006$. The system size was taken to be larger than $1500 d_{0} \times 3000 d_{0}$ which was determined from preliminary calculations. The steady-state value of each quantity was calculated by averaging over the time period of $t D_{l, 2} / d_{0}^{2}=$ $1.8 \times 10^{5}-2.0 \times 10^{5}$. Figure 5 represents the convergence behavior. Better convergence is achieved by the model with AT in comparison to that of the model without AT. The tip radius is relatively large in this case and the convergence of each quantity is fast. From the results of Figs. 2-5, one may roughly speculate that all the quantities are well converged as long as $W_{0} / \rho \sim 0.2$ is satisfied. This is consistent with the behavior observed in the previous study on the directional solidification of a dilute binary alloy [12].

As demonstrated above, reasonable convergence behavior is obtained by the present model and it suggests that one can utilize this model for quantitatively accurate simulations.

\section{SUMMARY}

In this study we have developed a quantitative phase-field model for nonisothermal solidification in multicomponent alloys with arbitrary diffusivities. We have given an exposition of the thin-interface asymptotics and simulated free dendritic growth into undercooled melt in binary and ternary alloys to check the performance of the model. Reasonable convergence behavior was demonstrated in each case. Hence, the present model is considered as a tool for quantitatively accurate and computationally efficient simulations of the solidification microstructure in the multicomponent system.

Let us conclude by remarking on some issues on further development of the quantitative modeling. First, our formulation was based on the dilute solution approximation. Although a number of practical alloys can be well described within this approximation, it is important to treat the systems with arbitrary thermodynamic properties in order to expand the application range of the model. In this regard, we would like to refer to several works $[15,45,52]$ that attempt to model arbitrary thermodynamic properties. Second, off-diagonal elements in the diffusivity matrix were not taken into account in this study, while these were explicitly dealt with in the previous work for one-sided model of multicomponent alloys [15]. As a step toward further generalization, it is important to include the off-diagonal elements in the diffusivity matrix. Last, this should be the most essential point regarding a correct thin-interface limit for the case of arbitrary diffusivities. At present, only two cases, the symmetric model and the one-sided model, are completely established based on the procedure of thin-interface limit. As long as Eq. (4.21) holds, the present model should be validated for the two-sided case.
However, this is the case for $V_{n} \neq 0$. If the static interface is considered, the contribution of antitrapping current disappears because it is proportional to $\partial_{t} \phi$ (thus, $V_{n}$ ). On the other hand, the anomalous interface effects associated with Eqs. (4.16) and (4.19) vanish, because their magnitudes are proportional to $V_{n}$. The effect associated with Eq. (4.20) can be eliminated by choosing an appropriate form for $q_{i}(\phi)$ as described in this paper. Therefore, the one-sided model is valid even for $V_{n}=$ 0 , since these are the only anomalous interface effects in this case. However, in the two-sided case, the effects related to Eqs. (4.17) and (4.18) still exist for $V_{n}=0$. The magnitudes of them are proportional not to $V_{n}$ but to the diffusion flux. In general, hence, the antitrapping current proportional to $\partial_{t} \phi$ cannot eliminate all the interface effects in the two-sided case [37]. This point remains as the most important task to be tackled for quantitative modeling. As mentioned, however, the present model is valid for $V_{n} \neq 0$, which is actually the case of great importance in most of studies on the microstructural evolution. In addition, the present model can be seen as a generalized model in that symmetric and one-sided models are reproduced as special cases. As demonstrated in this paper, above all, reasonable convergence behavior can be achieved by the present model, which strongly supports the validity and usefulness. We believe that the present model is one of the effective tools for simulating solidification microstructures in practical alloys.

\section{ACKNOWLEDGMENTS}

The author thanks Dr. Akinori Yamanaka at Tokyo University of Agriculture and Technology and Dr. Tomohiro Takaki at Kyoto Institute of Technology for providing valuable information about GPU computing. This work is partly supported by Grant-in Aid for Young Scientists (A) (No. 2268067) from MEXT, Japan. The author acknowledges the Strategic Programs for Innovative Research (SPIRE), MEXT, and the Computational Materials Science Initiative (CMSI), Japan.

\section{APPENDIX: VARIATIONAL FORMULATION OF THE PHASE-FIELD MODEL}

In this Appendix, we derive Eqs. (3.1)-(3.4) starting from the definition of free energy functional. As described in Sec. III, we introduce the phase field $\phi$ which takes $\phi=+1$ in the solid and $\phi=-1$ in the liquid and continuously changes from +1 to -1 inside the interface region. Following the KKS model [43], the interface region is defined as a mixture of the solid and liquid phases having the concentrations $\left\{c_{s, i}\right\}$ and $\left\{c_{l, i}\right\}$, respectively. Here the subscript $i$ specifies the type of atoms. The local concentration of $i$ atom is given by $c_{i}$ $=[1+g(\phi)] c_{s, i} / 2+[1-g(\phi)] c_{l, i} / 2$ with an interpolating function $g(\phi)$ that satisfies $g( \pm 1)= \pm 1$ and $g^{\prime}( \pm 1)=0$. Since the conservation of the internal energy is taken into account in this model, the entropy is a natural choice for the basic thermodynamic potential. However, we start from a free energy functional for expedience's sake. The Ginzburg-Landau-type free energy functional of the system is defined by

$$
F=\int_{v}\left[\frac{\sigma^{2}}{2}(\nabla \phi)^{2}+\omega f_{d w}(\phi)+f\left(T,\left\{c_{l, i}\right\},\left\{c_{s, i}\right\}\right)\right] d v
$$


where $\sigma$ and $\omega$ are constants, $f_{d w}(\phi)$ is a double-well potential with minima at $\phi= \pm 1$. The third term in the integrand $f$ is the bulk free energy density and it is given by $f=$ $[1+g(\phi)] f_{s}\left(T,\left\{c_{s, i}\right\}\right) / 2+[1-g(\phi)] f_{l}\left(T,\left\{c_{l, i}\right\}\right) / 2$ with the bulk free energy density of solid, $f_{s}\left(T,\left\{c_{s, i}\right\}\right)$, and liquid, $f_{l}\left(T,\left\{c_{l, i}\right\}\right)$. In the KKS model, the chemical potentials of the solid and liquid at each local point are taken to be the same, viz., $\partial f_{s} / \partial c_{s, i}=\partial f_{l} / \partial c_{l, i}=\mu_{i}$. It makes $c_{s, i}$ and $c_{l, i}$ mutually dependent. Taking into account this condition, one can obtain the temporal evolution equation of $\phi$,

$$
\begin{aligned}
\hat{M}_{\phi}^{-1} \partial_{t} \phi= & -\delta F / \delta \phi=\sigma^{2} \nabla^{2} \phi-\omega f_{d w}^{\prime} \\
& -\frac{1}{2} g^{\prime}(\phi)\left[f_{s}-f_{l}-\sum_{i}^{N-1} \mu_{i}\left(c_{s, i}-c_{l, i}\right)\right],
\end{aligned}
$$

where $\hat{M}_{\phi}$ is the phase-field mobility. The third term corresponds to the thermodynamic driving force and it vanishes when the solid and liquid concentrations are the equilibrium ones, that is, $c_{s, i}=c_{s, i}^{e}$ and $c_{l, i}=c_{l, i}^{e}$. Then, the interfacial energy (the excess free energy associated with the interface), $\gamma_{\text {int }}$, is determined by the counterbalance between the first and second terms. It is given by $\gamma_{\text {int }}=I W \omega$, where $W=\sigma / \omega^{1 / 2}$ is a measure of the interface thickness and $I$ is a numerical constant depending on the definition of $f_{d w}$. Importantly, the equilibrium phase-field profile in the interface region, the form of which is explicitly given once $f_{d w}$ is defined, depends only on $W$. Therefore, the spatial profile of $\phi$ and the interfacial energy $\gamma_{\text {int }}$ are independent of the concentration fields. This is the main advantage of the KKS model [43].

In this study, the bulk free energy density of $v$ phase $f_{v}$ is defined as follows:

$$
\begin{aligned}
f_{v}\left(T,\left\{c_{v, i}\right\}\right)= & f_{v, 0}(T) \\
& +\sum_{i}^{N-1}\left[\Omega_{v, i}(T) c_{v, i}+\frac{R T}{v_{m}}\left(c_{v, i} \ln c_{v, i}-c_{v, i}\right)\right],
\end{aligned}
$$

where $f_{v, 0}(T)$ is the free energy density of pure solvent, $v_{m}$ is the molar volume, and $R$ is the gas constant. The quantity $\Omega_{v, i}(T)$ is assumed as $\Omega_{v, i}(T)=\left(R T / v_{m}\right) \ln \hat{\gamma}_{v, i}$ with a constant $\hat{\gamma}_{v, i}$. Then, the condition of equal chemical potential, $\partial f_{s} / \partial c_{s, i}=\partial f_{l} / \partial c_{l, i}=\mu_{i}$, yields the relation, $c_{s, i}=k_{i} \mathrm{c}_{l, i}$, with the partition coefficient, $k_{i}=\hat{\gamma}_{l, i} / \hat{\gamma}_{s, i}$. By substituting Eq. (A3) into Eq. (A2), the thermodynamic driving force is expressed as

$$
\begin{aligned}
f_{s} & -f_{l}-\sum_{i=1}^{N-1} \mu_{i}\left(c_{s, i}-c_{l, i}\right) \\
& =f_{s, 0}(T)-f_{l, 0}(T)+\left(R T / v_{m}\right) \sum_{i=1}^{N-1}\left(1-k_{i}\right) c_{l, i} .
\end{aligned}
$$

When the temperature $T$ is close to $T_{m}$, the first and second terms can be approximated by $f_{s, 0}(T)-f_{l, 0}(T) \approx\left(L / T_{m}\right)$ $\left(T-T_{m}\right)$ and, furthermore, $T$ in the third term is approximated by $T_{m}$. When the equilibrium at $T=T_{0}\left(=T_{m}+\sum m_{i} c_{l, i}^{e}\right)$ is considered, viz., $c_{l, i}=c_{l, i}^{e}$, the right-hand side of Eq. (A4) should vanish because of no driving force in the equilibrium state. Then one finds the relation $m_{i}=-R T_{\mathrm{m}}^{2}\left(1-k_{i}\right) /\left(L v_{m}\right)$.
Using this relation, Eq. (A4) is rewritten as

$$
\begin{aligned}
& f_{s}-f_{l}-\sum_{i=1}^{N-1} \mu_{i}\left(c_{s, i}-c_{l, i}\right) \\
& \quad \approx\left(L / T_{m}\right)\left(T-T_{0}\right)+\left(R T_{m} / v_{m}\right) \sum_{i=1}^{N-1}\left(1-k_{i}\right)\left(c_{l, i}-c_{l, i}^{e}\right) .
\end{aligned}
$$

The time evolution of $\phi$ can be solved by substituting Eq. (A5) into Eq. (A2) and one can obtain Eq. (3.1) with Eq. (3.2).

The conservation law of solute atoms is described by $\partial_{t} c_{i}=$ $-\nabla \vec{J}_{c, i}$. The diffusion flux of $i$ atom $\vec{J}_{c, i}$ is defined as

$$
\vec{J}_{c, i}=-\hat{M}_{c, i}(\phi) \nabla\left(\mu_{i} / T\right)
$$

where $\hat{M}_{c, i}(\phi)$ is the mobility of $i$ atoms which is a function of $\phi$. The chemical potential in Eq. (A6) is obtained from Eq. (A3). When the condition of equal chemical potential is taken into account, the solute diffusion equation is expressed as

$$
\begin{aligned}
\partial_{t} c_{i}= & \nabla\left[\frac{1+g(\phi)}{2} \frac{R \hat{M}_{c, i}(\phi)}{v_{m} c_{s, i}} \nabla c_{s, i}\right. \\
& \left.+\frac{1-g(\phi)}{2} \frac{R \hat{M}_{c, i}(\phi)}{v_{m} c_{l, i}} \nabla c_{l, i}\right]=\nabla D_{i}(\phi) \nabla c_{l, i}
\end{aligned}
$$

where

$D_{i}(\phi)=\frac{1+g(\phi)}{2} \frac{k_{i} R \hat{M}_{c, i}(\phi)}{v_{m} c_{s, i}}+\frac{1-g(\phi)}{2} \frac{R \hat{M}_{c, i}(\phi)}{v_{m} c_{l, i}}$.

Here it is defined that $\hat{M}_{c, i}(\phi)$ satisfies the relations $R \hat{M}_{c, i}(+1) /\left(v_{m} c_{s, i}\right)=D_{s, i}, R \hat{M}_{c, i}(-1) /\left(v_{m} c_{l, i}\right)=D_{l, i}$, and thereby $D_{i}(+1)=k_{i} D_{s, i}$ and $D_{i}(-1)=D_{l, i}$. Although not explicitly specified for convenience, $D_{s, i}, D_{l, i}$, and thus $D_{i}(\phi)$ depend on the concentrations and temperature. The diffusion equation (A7) is identical to Eq. (3.3)

The conservation of energy is expressed by $\partial_{t} e=-\nabla \vec{J}_{T}$. The heat flux $\vec{J}_{T}$ is given by Fourier's law,

$$
\vec{J}_{T}=-\hat{M}_{T}(\phi) \nabla T
$$

where $\hat{M}_{T}(\phi)$ is the thermal conductivity of $\phi$-dependent. Using the bulk free energy [Eq. (A3)] and the thermodynamic relationships, $d e=T d s+\sum \mu_{i} d c_{i}$ and $s=-\partial f / \partial T$, one obtains the following equation:

$$
\partial_{t} T=\nabla\left[D_{T}(\phi) \nabla T\right]+\frac{L}{c_{p}} \frac{\partial_{t} g(\phi)}{2},
$$

where $D_{T}(\phi)=\hat{M}_{T}(\phi) / c_{p}$ is the thermal diffusivity which satisfies $D_{T}(+1)=D_{s, T}$ and $D_{T}(-1)=D_{l, T} \cdot c_{p}=$ $-T \partial^{2} f / \partial^{2} T$ is the heat capacity which are assumed to be equal in the solid and liquid. In the derivation of Eq. (A10), the approximation $T / T_{m} \sim 1$ was made in the second term on the right-hand side. This equation is equivalent to Eq. (3.4). 
[1] W. J. Boettinger, J. A. Warren, C. Beckermann, and A. Karma, Annu. Rev. Mater. Res. 32, 163 (2002).

[2] L. Gránásy, T. Pusztai, and J. A. Warren, J. Phys.: Condens. Matter 16, R1205 (2004).

[3] H. Emmerich, Adv. Phys. 57, 1 (2008).

[4] I. Steinbach, Model. Simul. Mater. Sci. Eng. 17, 073001 (2009).

[5] G. Caginalp, Phys. Rev. A 39, 5887 (1989).

[6] G. B. McFadden, A. A. Wheeler, R. J. Braun, S. R. Coriell, and R. F. Sekerka, Phys. Rev. E 48, 2016 (1993).

[7] J. J. Hoyt, M. Asta, and A. Karma, Mater. Sci. Eng., R 41, 121 (2003).

[8] A. Karma and W.-J. Rappel, Phys. Rev. E 53, R3017 (1996).

[9] A. Karma and W.-J. Rappel, Phys. Rev. E 57, 4323 (1998).

[10] R. F. Almgren, SIAM J Appl. Math. 59, 2086 (1999).

[11] A. Karma, Phy. Rev. Lett. 87, 115701 (2001).

[12] B. Echebarria, R. Folch, A. Karma, and M. Plapp, Phys. Rev. E 70, 061604 (2004).

[13] R. Folch and M. Plapp, Phys. Rev. E 72, 011602 (2005).

[14] J. C. Ramirez, C. Beckermann, A. Karma, and H.-J. Diepers, Phys. Rev. E 69, 051607 (2004).

[15] S. G. Kim, Acta Mater. 55, 4391 (2007).

[16] M. Greenwood, M. Haataja, and N. Provatas, Phys. Rev. Lett. 93, 246101 (2004).

[17] C. W. Lan and C. J. Shih, Phys. Rev. E 69, 031601 (2004).

[18] J. C. Ramirez and C. Beckermann, Acta Mater. 53, 1721 (2005).

[19] A. Badillo and C. Beckermann, Acta Mater. 54, 2015 (2006).

[20] H. Emmerich and R. Siquieri, J. Phys.: Condens. Matter 18, 11121 (2006).

[21] M. Plapp, J. Cryst. Growth 303, 49 (2007).

[22] R. Siquieri and H. Emmerich, Philos. Mag. Lett. 87, 829 (2007).

[23] A. Parisi and M. Plapp, Acta Mater. 56, 1348 (2008).

[24] J. Rosam, P. K. Jimack, and A. M. Mullis, Acta Mater. 56, 4559 (2008).

[25] I. Steinbach, Acta Mater. 56, 4965 (2008).

[26] P. K. Galenko, S. Reutzel, D. M. Herlach, S. G. Fries, I. Steinbach, and M. Apel, Acta Mater. 57, 6166 (2009).

[27] J. Rosam, P. K. Jimack, and A. M. Mullis, Phys. Rev. E 79, 030601(R) (2009).
[28] K. Oguchi and T. Suzuki, ISIJ Inter. 49, 1536 (2009).

[29] G. Boussinot, E. A. Brener, and D. E. Temkin, Acta Mater. 58, 1750 (2010)

[30] B. Echebarria, A. Karma, and S. Gurevich, Phys. Rev. E 81, 021608 (2010).

[31] S. Gurevich, A. Karma, M. Plapp, and R. Trivedi, Phys. Rev. E 81, 011603 (2010)

[32] A. M. Mullis, Phys. Rev. E 83, 061601 (2011).

[33] Z. Wang, J. Wang, J. Li, G. Yang, and Y. Zhou, Phys. Rev. E 84, 041604 (2011).

[34] J. Li, Z. Wang, Y. Wang, and J. Wang, Acta Mater. 60, 1478 (2012).

[35] A. Gopinath, R. C. Armstrong, and R. A. Brown, J. Cryst. Growth 291, 272 (2006).

[36] M. Ohno and K. Matsuura, Phys. Rev. E 79, 031603 (2009).

[37] M. Plapp, Philos. Mag. 91, 25 (2011).

[38] J. S. Langer, Rev. Mod. Phys. 52, 1 (1980).

[39] P.-R. Cha, D.-H. Yeon, and J.-K. Yoon, Acta Mater. 49, 3295 (2001).

[40] H. Kobayashi, M. Ode, S. G. Kim, and T. Suzuki, Scr. Mater. 48, 689 (2003).

[41] R. S. Qin and E. R. Wallach, Acta Mater. 51, 6199 (2003).

[42] J. Eiken, B. Böttger, and I. Steinbach, Phys. Rev. E 73, 066122 (2006).

[43] S. G. Kim, W. T. Kim, and T. Suzuki, Phys. Rev. E 60, 7186 (1999).

[44] J. Tiaden, B. Nestler, H.-J. Diepers, and I. Steinbach, Physica D 115, 73 (1998).

[45] M. Plapp, Phys. Rev. E 84, 031601 (2011).

[46] A. Choudhury and B. Nestler, Phys. Rev. E 85, 021602 (2012).

[47] G. B. McFadden, A. A. Wheeler, and D. M. Anderson, Physica D 144, 154 (2000).

[48] M. Nicoli, M. Plapp, and H. Henry, Phys. Rev. E 84, 046707 (2011).

[49] T. Haxhimali, A. Karma, F. Gonzales, and M. Rappaz, Nat. Mater. 5, 660 (2006).

[50] A. Yamanaka, T. Aoki, S. Ogawa, and T. Takaki, J. Cryst. Growth 318, 40 (2011).

[51] K. Glasner, J. Comput. Phys. 174, 695 (2001).

[52] C. Tong, M. Greenwood, and N. Provatas, Phys. Rev. B 77, 064112 (2008). 